\title{
CRYAB predicts clinical prognosis and is associated with immunocyte infiltration in colorectal cancer
}

\author{
Junsheng Deng ${ }^{\text {Equal first author, } 1}$, Xiaoli Chen ${ }^{\text {Equal first author, } 1, \text { Ting Zhan }^{1} \text {, Mengge Chen }}{ }^{2}$, Xisheng Yan ${ }^{3}$, Xiaodong Huang \\ Corresp. 2 \\ ${ }^{1}$ Gastroenterology, Tongren Hospital of Wuhan University, Wuhan, Hubei, China \\ 2 Gastroenterology, Tongren Hospital of Wuhan Unversity, Wuhan, Hubei, China \\ 3 Tongren Hospital of Wuhan Unversity, Wuhan, Hubei, China \\ Corresponding Author: Xiaodong Huang \\ Email address: 13297056720@163.com
}

Abstract. Background $\alpha B-C r y s t a l l i n(C R Y A B)$ is differentially expressed in various tumors. However, the correlation between CRYAB and immune cell infiltration in colorectal cancer remains unclear. Materials \& Methods CRYAB expression was examined in colorectal cancer (COAD) tissues and normal adjacent tissues from the Cancer Genome Atlas data and verified in a separate cohort from the Comprehensive Gene Expression database. Kaplan-Meier survival curves were used to evaluate the relationship between CRYAB expression and overall survival and progression-free survival. The relationship between CRYAB expression and infiltrating immune cells, and their corresponding gene marker sets was examined using the TIMER database. Results The expression of CRYAB was lower in COAD tumor tissues than in normal tissues $(P<0.05)$. High CRYAB gene expression and high levels of CRYAB gene methylation were associated with high-grade malignancy and advanced TNM stage. In addition, in colorectal cancer, there was a positive correlation between CRYAB expression and immune infiltrating cells, including neutrophils, macrophages, CD4 + T cells, and CD8 + T cells, as well as immune-related genes, including CD2, CD3D, and CD3E. Methylation sites such as cg13084335, cg15545878, cg13210534, and cg15318568 were positively correlated with low expression of CRYAB. Conclusion CRYAB may be a potential tumor suppressor gene in COAD and a potential novel therapeutic target and predictive biomarker for COAD. CRYAB likely plays an important role in immune cell infiltration. 
1 CRYAB predicts clinical prognosis and is associated with immunocyte infiltration in

2 colorectal cancer

3 Junsheng Deng ${ }^{\mathrm{a}, 1}$, Xiaoli Chen ${ }^{\mathrm{a} 1}$, Ting Zhan ${ }^{\mathrm{a}}$, Mengge Chen ${ }^{\mathrm{a}}$, Xisheng Yan ${ }^{\mathrm{a}}, \mathrm{Xiaodong}_{\mathrm{Huang}}^{\mathrm{a}}{ }^{*}$

4 a Department of Gastroenterology, Tongren Hospital of Wuhan University, Wuhan, 430060,

5 China

6 \# Corresponding author: Prof. Xiaodong Huang M.D, Tongren Hospital of Wuhan University,

7 No. 241, Pengliuyang Road, Wuchang District,Wuhan 430060, Hubei, China. E-mail:

$8 \quad 13297056720 @ 163 . c o m$

9 Abstract.

Background

$11 \alpha \mathrm{B}$-Crystallin (CRYAB) is differentially expressed in various tumors. However, the correlation between CRYAB and immune cell infiltration in colorectal cancer (CRC) remains unclear. Materials \& Methods

Kaplan-Meier survival curves in The Cancer Genome Atlas (TCGA) were used to evaluate the relationship between CRYAB expression and both overall survival and progression-free survival. The relationships between CRYAB expression and infiltrating immune cells and their corresponding gene marker sets were examined using the TIMER database.

\section{Results}

The expression of CRYAB was lower in CRC tumor tissues than in normal tissues $(P<0.05)$. High CRYAB gene expression and high levels of CRYAB gene methylation were correlated with high-grade malignant tumors and more advanced tumor, nodes and metastasis (TNM) cancer stages. In addition, in colorectal cancer, there was a positive correlation between CRYAB expression and immune infiltrating cells including neutrophils, macrophages, CD8 $+\mathrm{T}$ cells, and CD4 + T cells, as well as immune-related genes including CD2, CD3D, and CD3E. Methylation sites such as $\operatorname{cg} 13084335, \operatorname{cg} 15545878, \operatorname{cg} 13210534$, and $\operatorname{cg} 15318568$ were positively correlated with low expression of CRYAB. 
27

28

29

30

\section{Conclusion}

Because CRYAB likely plays an important role in immune cell infiltration, it may be a potential tumor-suppressor gene in CRC and a potential novel therapeutic target and predictive biomarker for colorectal cancer (CRC).

Keywords: CRYAB, colorectal cancer, immune cells, immune genes, methylation Introduction

Colorectal cancer (CRC) is one of the three most common cancers in the United States (Lurje et al., 2008; Lee et al., 2018) (after lung cancer, prostate cancer in men, and breast cancer in women) (Altun et al., 2013). According to estimates by the International Agency for Research on Cancer, in 2020, 1.9 million people worldwide were diagnosed (6\% of all cancer diagnoses) and 935,000 people died of CRC (5.8\% of all cancer-related deaths) (Sung et al., 2021). Approximately $28 \%$ of CRCs occur in the rectum, with $22 \%$ of these cases of CRC in the rectum involving the distal colorectal regions and 41\% involving the proximal colorectal regions (Cheng et al., 2011). Up to 50\% of CRC patients show metastatic disease at the time of diagnosis (Calon et al., 2012). Despite advances in screening and treatment options (Jeon et al., 2018), the 5-year survival rate of patients with metastatic disease is $<10 \%$ (Brenner, Kloor and Pox, 2014; De Stefano and Carlomagno, 2014; Suman et al., 2016). Biomarkers to help diagnose CRC early have yet to be identified, but personalized treatment strategies can improve the prognosis of patients with CRC (Yiu and Yiu, 2016). Increasing evidence supports the idea that malignant tumors depend on tumor cells and the tumor microenvironment, including extracellular matrix (ECM) molecules, inflammatory mediators, and immune cells (Hanahan and Coussens, 2012). Tumor-infiltrating immune cells are valuable for diagnosing cancer and identifying cancer progression and prognosis (Mlecnik et al., 2018; Kang et al., 2019; Zhou et al., 2019). The immune factors and immune cells that constitute the tumor immune microenvironment play a significant part in the progression and occurrence of anti-tumor immunity (Berraondo et al., 2016; Chen et al., 2017). Investigating the characteristics of tumor immune infiltration has been valuable for the treatment, evaluation, and diagnosis of many cancers (Peng et al., 2019; Zhang 
54

55

56

57

58

59

60

61

62

63

et al., 2019; Wei et al., 2020), and encouraging advances have been made in cancer immunotherapy in terms of treatment efficacy and long-term patient survival (Vonderheide, Domchek and Clark, 2017). Therefore, studying the tumor immune microenvironment of CRC is critical.

$\alpha \mathrm{B}$-Crystallin (CRYAB) has a C-terminal domain, an $\mathrm{N}$-terminal domain, and a central domain (Rajagopal et al., 2015). CRYAB may induce epithelial-mesenchymal transition (EMT) in CRC by activating the ERK signaling pathway, and can be an underlying cancer biomarker used in the prognosis and diagnosis of CRC (Li et al., 2017). In the nervous system, CRYAB plays a neuroprotective role in neurodegenerative diseases such as familial amyloidotic polyneuropathy, which is characterized by CRYAB overexpression (Magalhaes, Santos and Saraiva, 2010). However, no correlation has been found between CRYAB and immune cell infiltration. Chen et al. showed that CRYAB is correlated with poor prognosis in CRC (Shi et al., 2014) and promotes the invasion and metastasis of CRC via epithelial-mesenchymal transition (EMT) (Shi et al., 2017). However, the role of an underlying marker for the prognosis and diagnosis of CRC and the relationship between CRYAB and immune infiltration in CRC have not been investigated to date. In this study, various databases such as Gene Expression Omnibus (GEO) and The Cancer Genome Atlas (TCGA) were used to explore whether CRYAB expression level can be used as an indicator of poor prognosis and also explore the potential relation of CRYAB expression level and insufficient immune cell infiltration in CRC.

\section{Methods}

\section{Data source}

TCGA (https://genome-cancer.ucsc.edu/) provides scholars and researchers with clinical and pathological information on 33 types of cancer. Data of colorectal cancer(CRC) patients with RNA-Seq expression and matching clinicopathological information was obtained through the TCGA tool cancer browser. Because the database is publicly available and accessible, approval from the local ethics committee was not necessary. 
80

81

82

83

\section{GEO database}

The GEO database is a comprehensive gene expression library in the National Center for Biotechnology Information (NCBI) (https://www.ncbi.nlm.nih.gov/geo/) and is one of the largest collections of gene chips in the world.

\section{Immunochemistry}

Fresh colorectal cancer tissues and normal tissues were collected, immediately immersed in 4\% paraformaldehyde overnight, dehydrated by an ethanol series $(70 \%, 80 \%, 90 \%$, and $100 \%)$, clarified in xylene, and paraffin-embedded. They were subjected to a temperature of $60^{\circ} \mathrm{C}$ for 2 hours, deparaffinized, hydrated with xylene and ethanol, and then the recovered nuclear antigen was washed with PBS and hydrogen peroxide solution. Additional slices were randomly selected and incubated with rabbit anti-CRYAB antibody (\#15808-1-AP; Proteintech, Wuhan, China) at $4^{\circ} \mathrm{C}$ overnight, followed by HRP incubated goat anti-rabbit mouse universal antibody (1:3000, K5007, DAKO, Denmark) for 60 minutes at room temperature. After the slides were placed in PBS and decolorized, they were developed with DAB chromogenic solution. In addition, all slides (nuclei) were counterstained with $5 \mu \mathrm{g} / \mathrm{mL}$ Harris at room temperature for 3 minutes. Finally, the image was captured under a microscope (Nikon Eclipse E200; Tokyo, Japan).

\section{Statistical analysis of survival}

Patients in the experimental group and the validation groups were divided into two subgroups according to the median expression of the CRYAB gene: a high CRYAB expression group and a low CRYAB expression group. The effect of CRYAB expression level on the clinical outcomes of CRC patients was investigated using Kaplan-Meier (KM) survival curves, and a prognostic classifier was constructed to compare survival differences. The KM survival curve was implemented using the "survminer" package for analysis and visualization. 
103

104

105

106

107

108

109

110

111

112

113

114

115

116

117

118

119

120

121

122

123

124

125

126

\section{Analysis of CRYAB expression levels in colorectal cancer and normal colorectal samples}

To compare CRYAB expression patterns between tumor and normal tissues, the differential expression of CRYAB was examined in TCGA datasets using the Tumor Immunity Estimation Resource (TIMER) 2.0, a comprehensive online resource for the analysis of immune infiltrates and gene expression in different cancer types (Li et al., 2017; Li et al., 2020). In TIMER2.0, we selected "Exploration," then "Gene_De," followed by entering the gene name "CRYAB" in the text box and clicking "submit." Oncomine is a publicly accessible cancer gene chip database and web-based data mining platform containing 715 data sets and 86,733 samples (Rhodes et al., 2004; Rhodes et al., 2007). We searched the Oncomine server for human CRC, and chose the differential gene analysis segment (Normal Analysis vs. Cancer) to retrieve the results.

\section{TIMER database analysis}

The association between the expression of CRYAB and the presence of five infiltrating immune cells (neutrophils, macrophages, CD4 + T cells, CD8 + T cells) and three immunerelated genes (CD2, DC3D and CD3E) in CRC patients was evaluated using the TIMER database (http://timer.cistrome.org/).

\section{Meta-analysis}

A meta-analysis of data from the TCGA, GEO, and ICGC databases was performed to evaluate the significance of CRYAB expression in CRC prognosis. Heterogeneity among the included studies was determined by the $I^{2}$-value obtained from the Cochrane $Q$ test and the $P$ value obtained from the chi-square test. In cases of heterogeneity $\left(I^{2} \geq 50 \%\right.$ or $\left.P<0.05\right)$, the results were summarized using a random-effects model. Otherwise, a fixed-effect model was used for analysis. The "meta" R package (R version 4.0.0) was used to perform the metaanalysis.

\section{GO and KEGG enrichment analyses}


127 GO and KEGG enrichment analyses were performed using R 4.0.2 and the R packages "org. 128 Hs.eg.db," "ggplot2," "Cluster Profiler," and "enrich plot." Only terms with $P$-value $<0.05$ were 129 considered significantly enriched.

130

131

132

133

134

135

136

137

138

\section{Results}

\section{Patient characteristics}

The RNA sequencing data of 306 samples from the TCGA database and detailed clinical prognostic information were included in the analysis. Patients were divided into a low expression group $(n=153)$ and a high expression group $(n=153)$. Age, gender, and tumor size did not differ significantly between the high and low expression groups $(P=0.1695,0.6467,0.3418)$, however rates of lymphatic metastasis, metastasis and stage were significantly different between the high and low expression groups $(P=8.00 \mathrm{E}-04,0.0372,0.0101)$ (Table 1$)$.

\section{CRYAB expression is higher in normal tissues than in tumor samples}

The CRYAB gene is significantly downregulated in CRC (Fig. 1A). TIMER analysis of CRYAB expression in various cancer types showed that CRYAB expression was significantly lower in CRC tumor tissues than in normal tissues (Fig. 1B).

An analysis of the mRNA expression levels of CRYAB in TCGA samples showed that CRYAB expression was lower in tumor samples than in normal tissues $(P<2.22 \mathrm{e}-16)$ (Fig. 1C). This was verified in the GEO database $(P=0.045)$ (Fig. 1F). A meta-analysis of the above three datasets was performed to evaluate the correlation between overall survival (OS) and CRYAB gene expression and to obtain more objective conclusions. Because there was no statistically significant difference between the three datasets $\left(P=0.55, \mathrm{I}^{2}=0 \%\right)$, a fixed effects model was used to evaluate the combined hazard ratio (HR) and 95\% confidence interval (CI). A relatively high expression of the CRYAB gene was significantly correlated with poor OS $(\mathrm{HR}=1.23,95 \%$ CI: $1.11-1.35, P<0.0001$; Fig. $1 \mathrm{G})$, indicating that CRYAB may be a predictor of poor OS.

\section{Higher CRYAB mRNA expression in CRC is associated with shorter OS}

According to the $\mathrm{KM}$ chart, $\mathrm{CRC}$ cases with higher CRYAB mRNA expression had a shorter OS $(P=0.027)$ (Fig. 1D) and progression-free survival $(P=0.027)$ in the test cohort (Fig. 
154

155

156

157

158

159

160

161

162

163

164

165

166

167

168

169

170

171

172

173

174

175

176

177

178

179

180

1E). The results showed that the expression of CRYAB was lower in colorectal tumors than in normal colorectal tissues.

\section{Colorectal cancer CRYAB expression analysis}

The expression of CRYAB in CRC was confirmed by immunohistochemistry (Fig. 2A-F). The results confirmed that CRYAB expression was lower in CRC tissues than in normal colorectal tissues. We cut three sets of immunohistochemical slices, measured the positive rate of CRYAB among them, and drew the corresponding histogram. the positive rate of CRYAB was higher in tissue adjacent to tumors than in actual cancer tissue $(p<0.0001)$ (Fig. 2G).

\section{Correlation between CRYAB expression and clinical characteristics}

We analyzed the association between CRYAB mRNA expression and clinicopathological parameters in $\mathrm{CRC}$ patients and found that $\mathrm{CRYAB}$ level was not correlated with gender $(P=$ 0.63) (Fig. 3B). We also found that CRYAB expression decreased with age $(P=0.026)$ (Fig. $3 \mathrm{~A})$, and that increased CRYAB expression was associated with distant metastasis from CRC (Fig. 3C). Low expression of CRYAB was associated with colorectal cancer progression from N0 to N1 and from N0 to N2 $(P<0.001,0.01)$ (Fig. 3D). Low expression of CRYAB was also associated with CRC tumor size progression from T2 to T3, with an even stronger association of low CRYAB expression with the progression from T2 to T4 $(P=0.07,0.02)$. In terms of lymph node metastasis, the expression level of CRYAB did not change after N1 (Fig. 3E). High CRYAB expression was associated with tumor progression from Stage I to III, from Stage I to IV, from Stage II to II, and from Stage II to IV $(P<0.001,0.001,0.05,0.01)$. The level of CRYAB gene methylation was not associated with age, sex, TNM stage, or tumor stage (Fig. $3 \mathrm{G}-\mathrm{L})$.

\section{Correlation analysis between infiltrating immune cells and CRYAB expression}

Tumor infiltrating lymphocytes impact cancer survival(Mou et al., 2021). Therefore, we analyzed the correlation between CRYAB expression and four infiltrating immune cells (neutrophils, macrophages, CD8 + T cells, and CD4 + T cells) and three immune-related genes $(\mathrm{CD} 2, \mathrm{CD} 3 \mathrm{D}$, and CD3E). The results showed that the expression level of CRYAB is 
181

182

183

184

185

186

187

188

189

190

191

192

193

194

195

196

197

198

199

200

201

202

203

204

205

206

comparable to neutrophils $(\mathrm{r}=0.364, P=4.6 \mathrm{e}-10)$, macrophages $(\mathrm{r}=0.515, P=4.23 \mathrm{e}-20)$, CD4+ T cells $(\mathrm{r}=0.321, P=4.99 \mathrm{e}-08), \mathrm{CD} 8+\mathrm{T}$ cells $(\mathrm{r}=0.134, P=2.6 \mathrm{e}-02), \mathrm{CD} 2(\mathrm{r}=0.176$, $P=1.49$ e-04), CD3D (r0.176, $\mathrm{r}=P=1.12 \mathrm{e}-02)$ and CD3E $(\mathrm{r}=0.209, P=6.45 \mathrm{e}-06)(2021.5 .9)$ and that infiltration levels were significantly positively correlated with CRYAB expression with significance defined as $P<0.05$ (Fig. 4). In addition, CRYAB expression was significantly correlated with markers of M2-like macrophages in pan-carcinoma and CRC, including TGFB1 (Fig. 5A and B), MRC1 (Fig. 5C and D), and CD163 (Fig. 5E and F). These results indicate that in addition to the immunosuppressive microenvironment of colorectal cancer, high CRYAB expression was related to macrophage infiltration and polarization.

\section{DNA methylation analysis}

The degree of methylation of cg12598198 was the highest, followed from high to low by cg14276286, cg15227610, cg1158277, cg15204861, cg12947833, cg15318568, cg00514609, cg07476508, cg10048349, cg13210534, cg15545878, and cg13084335 (Fig. 6A). Among them, methylation sites such as $\operatorname{cg} 13084335, \operatorname{cg} 15545878, \operatorname{cg} 13210534$, and $\operatorname{cg} 15318568$ were positively correlated with low expression of CRYAB. The sites cg12598198, cg14276286, cg15227610, cg1158277, cg15204861, cg12947833, cg00514609, cg07476508, and cg10048349 and other methylation sites were negatively correlated with low expression of CRYAB (Fig. 6BO). The methylation level of CRYAB was lower in CRC than in the normal control group, indicating that changes in methylation are related to the abnormal expression of CRYAB. In addition, DNA methylation may be related to the molecular mechanism underlying the low expression level of CRYAB in tumor tissues and also to the pathogenesis of CRC.

\section{GO and KEGG enrichment analyses}

We performed a GO enrichment analysis and a KEGG pathway analysis. Figure 3A shows the top 30 significantly enriched upregulated pathways. The GO pathway analysis showed that relatively high expression of CRYAB was associated with extracellular structure organization (GO:0043062, $P=2.13 \mathrm{E}-64)$, ECM organization (GO:0030198, $P=1.76 \mathrm{E}-64)$, collagen- 
207

208

209

210

211

212

213

214

215

216

217

218

219

220

221

222

223

224

225

226

227

228

229

230

231

232

233

containing ECM (GO:0062023, $P=3.59 \mathrm{E}-85)$, and ECM structural constituent (GO:0005201, $P$

$=3.86 \mathrm{E}-70)$ (Fig. 7A). The KEGG pathway analysis identified many enriched pathways, including PI3K-Akt signaling pathway, focal adhesion, human papillomavirus infection, ECMreceptor interaction, and protein digestion and absorption, and that CRYAB genes were strongly linked to them(Fig. 7B)

\section{Discussion}

CRYAB is a principal member of the small molecule heat shock protein family (Annertz et al., 2014). CRYAB functions primarily as a molecular chaperone, preventing other proteins from stress injury, including those caused by heat shock, radiation, and oxidative stress (Moyano et al., 2006). Research shows that CRYAB can promote the occurrence and development of tumors (Li et al., 2017; Shi et al., 2017). M2 macrophages promote NSCLC metastasis by upregulating CRYAB (Guo et al., 2019). We found that CRYAB is downregulated in CRC, which is contrary to the results of previous studies (Shi et al., 2014). The gene expression in different tumors was inconsistent, which may be related to the specific environment (Dey et al., 2021), because gene expression is affected by many factors, such as the expression of PDCD1 in different tumors. PDCD1 expression is induced by TCR and/or B-cell receptor signaling, but this expression is frequently enhanced by tumor necrosis factor (TNF) stimulation (Nakae et al., 2006). The underlying mechanism may involve different patterns of CRYAB phosphorylation in different tumors, which determine the protein-binding library and biological effects of each tumor (Kuipers et al., 2017).

We identified CRYAB as a new potential therapeutic target and predictive biomarker for $\mathrm{CRC}$ and found that CRYAB can be used as a prognostic indicator of immune status. We also found that relatively high expression of $C R Y A B$ and the methylation of CRYAB in tumor tissues are both related to the infiltration of immune cells. The methylation level of CRYAB was not related to age, gender, TNM stage, or tumor stage in our analyses.

The KEGG pathway analysis and GO functional enrichment analysis showed that CRYAB was significantly associated with tumorigenesis and tumor development pathways, including: 
234 focal adhesion for cell migration (Paluch, Aspalter and Sixt, 2016), the PI3K-AKT signaling 235 pathway, ECM organization, human papillomavirus infection leading to cervical cancer

236 (Schiffman et al., 2016), and ECM-receptor interaction leading to melanoma metastasis (Chen et 237 al., 2019). The intestinal ECM is mainly composed of collagen, which is essential for regulating

238

239

240

241

242

243

244

245

246

247

248

249

250

251

252

253

254

255

256

257

258

259

260 cell division, differentiation, proliferation, growth, migration, and apoptosis indicating that it plays a vital part in the development and progression of cancer (Fischer et al., 2001). Therefore, this indicates that the high expression of CRYAB is involved in the positive regulation of these signal pathways. CRYAB may play a pathological role in promoting tumor cell proliferation by driving the up-regulation of these signal transduction pathways. In addition, CRYAB may inhibit tumor cell migration and invasion through overexpression in T24 and J82 BC cell lines(Ruan et al., 2020). The high expression of CRYAB could promote the proliferation, invasion, and metastasis of CRC through EMT(Zhang et al., 2019). Its expression level in CRC patients is closely related to the two core EMT gene products, MMP7 and E-cadherin. Furthermore, three important signaling pathways (PI3K, p38, and ERK) are involved in CRYAB-induced EMT(Worthley and Leggett, 2010; Giordano et al., 2015; Li et al., 2017). Hence, although CRYAB can be used as a tumor-suppressor gene in CRC, the expression of CRYAB in highly malignant cancers is increased. We used the TIMER database to reveal, for the first time, that the expression of CRYAB in CRC is associated with the infiltration of a variety of immune cells.

Tumor-infiltrating lymphocytes, such as tumor correlated macrophages and cancer-infiltrating neutrophils, may affect the prognosis and efficacy of chemotherapy and immunotherapy (Waniczek et al., 2017; Zhang et al., 2018). The level of tumor infiltration by immune cells is correlated with tumor growth, progression, and patient outcome (Gajewski, Schreiber and Fu, 2013). A close relationship between immune infiltration and the occurrence and development of CRC was reported previously (Xiong et al., 2018), however, there were no studies analyzing the relationship between CRYAB expression and immune cell infiltration. We evaluated the relationship between CRYAB expression and the immune infiltration level of CRC using the TIMER website. CD2, CD3D, and CD3E genes were positively related to the infiltration of 
261

262

263

264

265

266

267

268

269

270

271

272

273

274

275

276

277

278

279

280

281

282

283

284

285

286

287

288

289

\section{4} (ats

neutrophils, macrophages, CD8 + T cells, CD4 + T cells, and CD2, CD3D, and CD3E genes. Our findings confirm that the increased infiltration levels of immune cells are crucial for the progression of CRC, and CRYAB expression is a predictor of that increase of immune cell infiltration. These results indicate the need for further research on the relationship between CRYAB and CRC immune infiltration.

The present study had several limitations. We only obtained data from TCGA, including mRNA expression and methylated expression, which may lead to data deviation in this study. A larger number of tumor specimens and further experimental verification are needed to evaluate the biological role of CRYAB in CRC. In addition, further experimental validation is needed to determine the value of CRYAB for predicting the prognosis of CRC and for use in the formulation of treatment strategies.

\section{Conclusion}

The present study provides important evidence supporting the importance of CRYAB in the prognosis of human CRC. We identified CRYAB as a novel biomarker and clarified its prognostic potential in $\mathrm{CRC}$ through the analysis of online public databases. Its biological function and role in immune infiltration were examined to elucidate the mechanism underlying its relatively high expression, which is the basis of CRC. Our results indicate that CRYAB can be used as a potential tumor suppressor gene in CRC. CRYAB may also be a new potential therapeutic target and predictive biomarker for CRC. Our experimental data provides insights that can be used for the development of appropriate treatment strategies and to further research on the topic. In addition, we are committed to studying cancer cell lines and mouse models of $\mathrm{CRC}$ to validate the present findings and develop effective treatment strategies by targeting the CRYAB gene.

Altun, A., T. K. Temiz, E. Balci, Z. A. Polat and M. Turan(2013). "Effects of tyrosine kinase inhibitor E7080 and eNOS inhibitor L-NIO on colorectal cancer alone and in combination." Chin J Cancer Res 25(5): 572-84.

Annertz, K., J. Enoksson, R. Williams, H. Jacobsson, W. B. Coman and J. Wennerberg(2014). "Alpha B-crystallin a validated prognostic factor for poor prognosis in squamous cell carcinoma of the oral cavity." Acta Otolaryngol 
290

291

292

293

294

295

296

297

298

299

300

301

302

303

304

305

306

307

308

309

310

311

312

313

314

315

316

317

318

319

320

321

322

323

324

325

326

327

328

329

330

134(5): 543-50.

Berraondo, P., L. Minute, D. Ajona, L. Corrales, I. Melero and R. Pio(2016). "Innate immune mediators in cancer: between defense and resistance." Immunol Rev 274(1): 290-306.

Brenner, H., M. Kloor and C. P. Pox(2014). "Colorectal cancer." Lancet 383(9927): 1490-1502.

Calon, A., E. Espinet, S. Palomo-Ponce, D. V. Tauriello, M. Iglesias, M. V. Cespedes, M. Sevillano, C. Nadal, P. Jung, X. H. Zhang, D. Byrom, A. Riera, D. Rossell, R. Mangues, J. Massague, E. Sancho and E. Batlle(2012). "Dependency of colorectal cancer on a TGF-beta-driven program in stromal cells for metastasis initiation." Cancer Cell 22(5): 571-84.

Chen, J., F. Wu, Y. Shi, D. Yang, M. Xu, Y. Lai and Y. Liu(2019). "Identification of key candidate genes involved in melanoma metastasis." Mol Med Rep 20(2): 903-914.

Chen, Y. P., Y. Zhang, J. W. Lv, Y. Q. Li, Y. Q. Wang, Q. M. He, X. J. Yang, Y. Sun, Y. P. Mao, J. P. Yun, N. Liu and J. Ma(2017). "Genomic Analysis of Tumor Microenvironment Immune Types across 14 Solid Cancer Types: Immunotherapeutic Implications." Theranostics 7(14): 3585-3594.

Cheng, L., C. Eng, L. Z. Nieman, A. S. Kapadia and Du XL(2011). "Trends in colorectal cancer incidence by anatomic site and disease stage in the United States from 1976 to 2005." Am J Clin Oncol 34(6): 573-80.

De Stefano, A. and C. Carlomagno(2014). "Beyond KRAS: Predictive factors of the efficacy of anti-EGFR monoclonal antibodies in the treatment of metastatic colorectal cancer." World J Gastroenterol 20(29): 9732-43.

Dey, A., S. Uppal, J. Giri and H. S. Misra(2021). "Emerging roles of Bromodomain protein 4 in regulation of stem cell identity." Stem Cells.

Fischer, H., R. Stenling, C. Rubio and A. Lindblom(2001). "Colorectal carcinogenesis is associated with stromal expression of COL11A1 and COL5A2." Carcinogenesis 22(6): 875-8.

Gajewski, T. F., H. Schreiber and Y. X. Fu(2013). "Innate and adaptive immune cells in the tumor microenvironment." Nat Immunol 14(10): 1014-22.

Giordano, G., A. Febbraro, E. Tomaselli, M. L. Sarnicola, P. Parcesepe, D. Parente, N. Forte, A. Fabozzi, A. Remo, A. Bonetti, E. Manfrin, S. Ghasemi, M. Ceccarelli, L. Cerulo, F. Bazzoni and M. Pancione(2015). "Cancer-related CD15/FUT4 overexpression decreases benefit to agents targeting EGFR or VEGF acting as a novel RAF-MEK-ERK kinase downstream regulator in metastatic colorectal cancer." J Exp Clin Cancer Res 34: 108.

Guo, Z., J. Song, J. Hao, H. Zhao, Du X, E. Li, Y. Kuang, F. Yang, W. Wang, J. Deng and Q. Wang(2019). "M2 macrophages promote NSCLC metastasis by upregulating CRYAB." Cell Death Dis 10(6): 377.

Hanahan, D. and L. M. Coussens(2012). "Accessories to the crime: functions of cells recruited to the tumor microenvironment." Cancer Cell 21(3): 309-22.

Jeon, J., Du M, R. E. Schoen, M. Hoffmeister, P. A. Newcomb, S. I. Berndt, B. Caan, P. T. Campbell, A. T. Chan, J. Chang-Claude, G. G. Giles, J. Gong, T. A. Harrison, J. R. Huyghe, E. J. Jacobs, L. Li, Y. Lin, L. Le Marchand, J. D. Potter, C. Qu, S. A. Bien, N. Zubair, R. J. Macinnis, D. D. Buchanan, J. L. Hopper, Y. Cao, R. Nishihara, G. Rennert, M. L. Slattery, D. C. Thomas, M. O. Woods, R. L. Prentice, S. B. Gruber, Y. Zheng, H. Brenner, R. B. Hayes, E. White, U. Peters and L. Hsu(2018). "Determining Risk of Colorectal Cancer and Starting Age of Screening Based on Lifestyle, Environmental, and Genetic Factors." Gastroenterology 154(8): 2152-2164.e19.

Kang, H. J., J. H. Oh, S. M. Chun, D. Kim, Y. M. Ryu, H. S. Hwang, S. Y. Kim, J. An, E. J. Cho, H. Lee, J. H. Shim, C. O. Sung and E. Yu(2019). "Immunogenomic landscape of hepatocellular carcinoma with immune cell stroma and EBV-positive tumor-infiltrating lymphocytes." J Hepatol 71(1): 91-103.

Kuipers, H. F., J. Yoon, J. van Horssen, M. H. Han, P. L. Bollyky, T. D. Palmer and L. Steinman(2017). 
331

332

333

334

335

336

337

338

339

340

341

342

343

344

345

346

347

348

349

350

351

352

353

354

355

356

357

358

359

360

361

362

363

364

365

366

367

368

369

370

371

"Phosphorylation of alphaB-crystallin supports reactive astrogliosis in demyelination." Proc Natl Acad Sci U S A 114(9): E1745-E1754.

Lee, G., J. Y. Joung, J. H. Cho, C. G. Son and N. Lee(2018). "Overcoming P-Glycoprotein-Mediated Multidrug Resistance in Colorectal Cancer: Potential Reversal Agents among Herbal Medicines." Evid Based Complement Alternat Med 2018: 3412074.

Li, Q., Y. Wang, Y. Lai, P. Xu and Z. Yang(2017). "HspB5 correlates with poor prognosis in colorectal cancer and prompts epithelial-mesenchymal transition through ERK signaling." PLoS One 12(8): e0182588.

Li, Q., Y. Wang, Y. Lai, P. Xu and Z. Yang(2017). "HspB5 correlates with poor prognosis in colorectal cancer and prompts epithelial-mesenchymal transition through ERK signaling." PLoS One 12(8): e0182588.

Li, Q., Y. Wang, Y. Lai, P. Xu and Z. Yang(2017). "HspB5 correlates with poor prognosis in colorectal cancer and prompts epithelial-mesenchymal transition through ERK signaling." PLoS One 12(8): e0182588.

Li, T., J. Fan, B. Wang, N. Traugh, Q. Chen, J. S. Liu, B. Li and X. S. Liu(2017). "TIMER: A Web Server for Comprehensive Analysis of Tumor-Infiltrating Immune Cells." Cancer Res 77(21): e108-e110.

Li, T., J. Fu, Z. Zeng, D. Cohen, J. Li, Q. Chen, B. Li and X. S. Liu(2020). "TIMER2.0 for analysis of tumor-infiltrating immune cells." Nucleic Acids Res 48(W1): W509-W514.

Lurje, G., W. Zhang, A. M. Schultheis, D. Yang, S. Groshen, A. E. Hendifar, H. Husain, M. A. Gordon, F. Nagashima, H. M. Chang and H. J. Lenz(2008). "Polymorphisms in VEGF and IL-8 predict tumor recurrence in stage III colon cancer." Ann Oncol 19(10): 1734-41.

Magalhaes, J., S. D. Santos and M. J. Saraiva(2010). "alphaB-crystallin (HspB5) in familial amyloidotic polyneuropathy." Int J Exp Pathol 91(6): 515-21.

Mlecnik, B., M. Van den Eynde, G. Bindea, S. E. Church, A. Vasaturo, T. Fredriksen, L. Lafontaine, N. Haicheur, F. Marliot, D. Debetancourt, G. Pairet, A. Jouret-Mourin, J. F. Gigot, C. Hubert, E. Danse, C. Dragean, J. Carrasco, Y. Humblet, V. Valge-Archer, A. Berger, F. Pages, J. P. Machiels and J. Galon(2018). "Comprehensive Intrametastatic Immune Quantification and Major Impact of Immunoscore on Survival." J Natl Cancer Inst 110(1).

Mou, Y., J. Wu, Y. Zhang, O. Abdihamid, C. Duan and B. Li(2021). "Low expression of ferritinophagy-related NCOA4 gene in relation to unfavorable outcome and defective immune cells infiltration in clear cell renal carcinoma." BMC Cancer 21(1): 18.

Moyano, J. V., J. R. Evans, F. Chen, M. Lu, M. E. Werner, F. Yehiely, L. K. Diaz, D. Turbin, G. Karaca, E. Wiley, T. O. Nielsen, C. M. Perou and V. L. Cryns(2006). "AlphaB-crystallin is a novel oncoprotein that predicts poor clinical outcome in breast cancer." J Clin Invest 116(1): 261-70.

Nakae, S., H. Suto, M. Iikura, M. Kakurai, J. D. Sedgwick, M. Tsai and S. J. Galli(2006). "Mast cells enhance T cell activation: importance of mast cell costimulatory molecules and secreted TNF." J Immunol 176(4): 2238-48.

Paluch, E. K., I. M. Aspalter and M. Sixt(2016). "Focal Adhesion-Independent Cell Migration." Annu Rev Cell Dev Biol 32: 469-490.

Peng, D., L. Wang, H. Li, C. Cai, Y. Tan, B. Xu and Le H(2019). "An immune infiltration signature to predict the overall survival of patients with colon cancer." IUBMB Life 71(11): 1760-1770.

Rajagopal, P., E. Tse, A. J. Borst, S. P. Delbecq, L. Shi, D. R. Southworth and R. E. Klevit(2015). "A conserved histidine modulates HSPB5 structure to trigger chaperone activity in response to stress-related acidosis." Elife 4.

Rhodes, D. R., J. Yu, K. Shanker, N. Deshpande, R. Varambally, D. Ghosh, T. Barrette, A. Pandey and A. M. Chinnaiyan(2004). "ONCOMINE: a cancer microarray database and integrated data-mining platform." Neoplasia 6(1): $1-6$. 
372 Rhodes, D. R., S. Kalyana-Sundaram, V. Mahavisno, R. Varambally, J. Yu, B. B. Briggs, T. R. Barrette, M. J. Anstet,

373 C. Kincead-Beal, P. Kulkarni, S. Varambally, D. Ghosh and A. M. Chinnaiyan(2007). "Oncomine 3.0: genes, 374 pathways, and networks in a collection of 18,000 cancer gene expression profiles." Neoplasia 9(2): 166-80.

375 Ruan, H., Y. Li, X. Wang, B. Sun, W. Fang, S. Jiang and C. Liang(2020). "CRYAB inhibits migration and invasion 376 of bladder cancer cells through the PI3K/AKT and ERK pathways." Jpn J Clin Oncol 50(3): 254-260.

377 Schiffman, M., J. Doorbar, N. Wentzensen, S. de Sanjose, C. Fakhry, B. J. Monk, M. A. Stanley and S. 378 Franceschi(2016). "Carcinogenic human papillomavirus infection." Nat Rev Dis Primers 2: 16086.

379 Shi, C., X. Yang, X. Bu, N. Hou and P. Chen(2017). "Alpha B-crystallin promotes the invasion and metastasis of 380 colorectal cancer via epithelial-mesenchymal transition." Biochem Biophys Res Commun 489(4): $369-374$.

381

382

383

384

385

386

387

388

389

390

391

392

393

394

395

396

397

398

399

400

401

402

403

404

405

406

407

408

409

410

411

412
Shi, C., X. Yang, X. Bu, N. Hou and P. Chen(2017). "Alpha B-crystallin promotes the invasion and metastasis of colorectal cancer via epithelial-mesenchymal transition." Biochem Biophys Res Commun 489(4): 369-374.

Shi, C., Z. He, N. Hou, Y. Ni, L. Xiong and P. Chen(2014). "Alpha B-crystallin correlates with poor survival in colorectal cancer." Int J Clin Exp Pathol 7(9): 6056-63.

Suman, S., T. P. Das, S. Sirimulla, H. Alatassi, M. K. Ankem and C. Damodaran(2016). "Withaferin-A suppress AKT induced tumor growth in colorectal cancer cells." Oncotarget 7(12): 13854-64.

Sung, H., J. Ferlay, R. L. Siegel, M. Laversanne, I. Soerjomataram, A. Jemal and F. Bray(2021). "Global cancer statistics 2020: GLOBOCAN estimates of incidence and mortality worldwide for 36 cancers in 185 countries." $\underline{\text { CA }}$ Cancer J Clin.

Vonderheide, R. H., S. M. Domchek and A. S. Clark(2017). "Immunotherapy for Breast Cancer: What Are We Missing?" Clin Cancer Res 23(11): 2640-2646.

Waniczek, D., Z. Lorenc, M. Snietura, M. Wesecki, A. Kopec and M. Muc-Wierzgon(2017). "Tumor-Associated Macrophages and Regulatory T Cells Infiltration and the Clinical Outcome in Colorectal Cancer." Arch Immunol Ther Exp (Warsz) 65(5): 445-454.

Wei, Z. W., J. Wu, W. B. Huang, J. Li, X. F. Lu, Y. J. Yuan, W. J. Xiong, X. H. Zhang, W. Wang, Y. L. He and C. H. Zhang(2020). "Immune-infiltration based signature as a novel prognostic biomarker in gastrointestinal stromal tumour." EBioMedicine 57: 102850.

Worthley, D. L. and B. A. Leggett(2010). "Colorectal cancer: molecular features and clinical opportunities." $\underline{\text { Clin }}$ Biochem Rev 31(2): 31-8.

Xiong, Y., K. Wang, H. Zhou, L. Peng, W. You and Z. Fu(2018). "Profiles of immune infiltration in colorectal cancer and their clinical significant: A gene expression-based study." Cancer Med 7(9): 4496-4508.

Yiu, A. J. and C. Y. Yiu(2016). "Biomarkers in Colorectal Cancer." Anticancer Res 36(3): 1093-102.

Zhang, B., Q. Wang, C. Fu, C. Jiang and S. Ma(2019). "Exploration of the immune-related signature and immune infiltration analysis for breast ductal and lobular carcinoma." Ann Transl Med 7(23): 730.

Zhang, H., H. Liu, Z. Shen, C. Lin, X. Wang, J. Qin, X. Qin, J. Xu and Y. Sun(2018). "Tumor-infiltrating Neutrophils is Prognostic and Predictive for Postoperative Adjuvant Chemotherapy Benefit in Patients With Gastric Cancer." Ann Surg 267(2): 311-318.

Zhang, J., J. Liu, J. Wu, W. Li, Z. Chen and L. Yang(2019). "Progression of the role of CRYAB in signaling pathways and cancers." Onco Targets Ther 12: 4129-4139.

Zhou, R., J. Zhang, D. Zeng, H. Sun, X. Rong, M. Shi, J. Bin, Y. Liao and W. Liao(2019). "Immune cell infiltration as a biomarker for the diagnosis and prognosis of stage I-III colon cancer." Cancer Immunol Immunother $\mathbf{6 8}(3)$ : 433-442. 


\section{Table $\mathbf{1}$ (on next page)}

Correlation between CRYAB expression in tumor and clinicopathological characteristics of patients with colorectal cancer

Correlation between CRYAB expression in tumor and clinicopathological characteristics of patients with colorectal cancer 


\section{Table Legends:}

2 Table 1 Correlation between CRYAB expression in tumor and clinicopathological

3 characteristics of patients with colorectal neoplasms

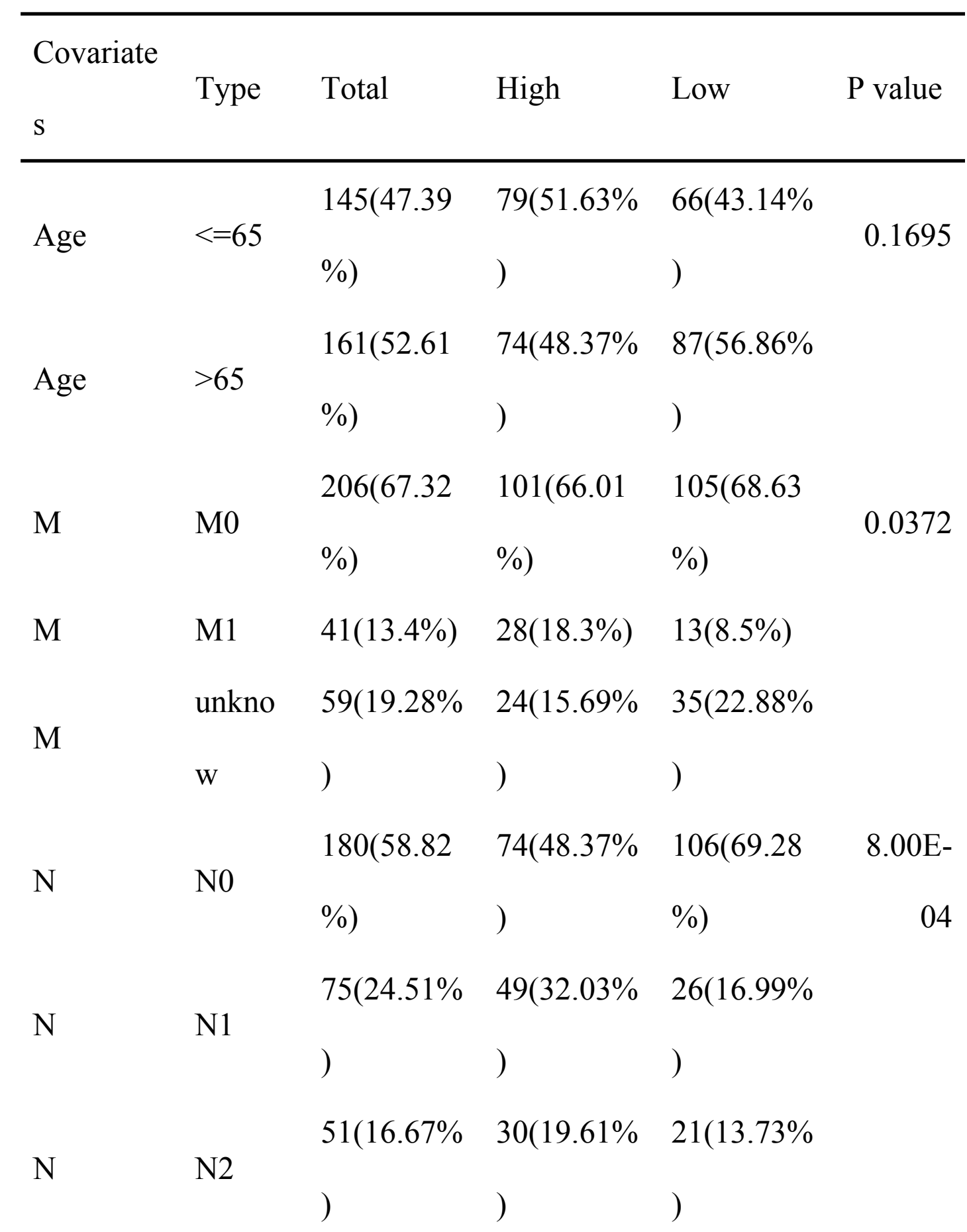




$\begin{array}{llllll}\mathrm{T} & \mathrm{T} 1 & 7(2.29 \%) & 3(1.96 \%) & 4(2.61 \%) & 0.3418 \\ \mathrm{~T} & \mathrm{~T} 2 & 46(15.03 \% & 18(11.76 \% & \\ & & & & 28(18.3 \%) \\ \mathrm{T} & \mathrm{T} 3 & 210(68.63 & 108(70.59 & 102(66.67 \\ & & \%) & \%) & \%) \\ \mathrm{T} & \mathrm{T} 4 & 42(13.73 \% & 24(15.69 \% & 18(11.76 \% \\ & & & & \end{array}$

$\mathrm{T}$

unkno

w
$1(0.33 \%)$
$1(0.65 \%)$
$143(46.73 \quad 74(48.37 \%$
Gender female
$69(45.1 \%)$
0.6467
$\%)$
)

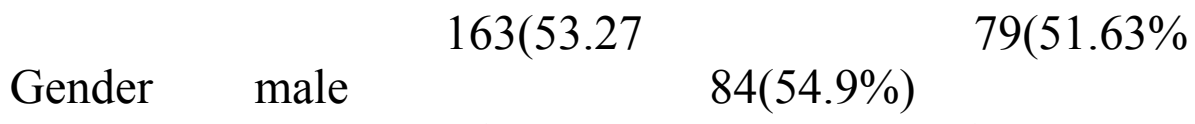
$\%) \quad$ )
$\begin{array}{llllll} & & 47(15.36 \% & 18(11.76 \% & 29(18.95 \% & \\ \text { Stage } & \text { Stage I } & & & 0.0101\end{array}$
$120(39.22 \quad 53(34.64 \% \quad 67(43.79 \%$

Stage Stage II

$\begin{array}{llll} & \%) & & \\ \text { Stage } & 88(28.76 \% & 50(32.68 \% & 38(24.84 \%\end{array}$

Stage

III ） ） （

Stage $\quad$ Stage $\quad 41(13.4 \%) \quad 28(18.3 \%) \quad 13(8.5 \%)$ 


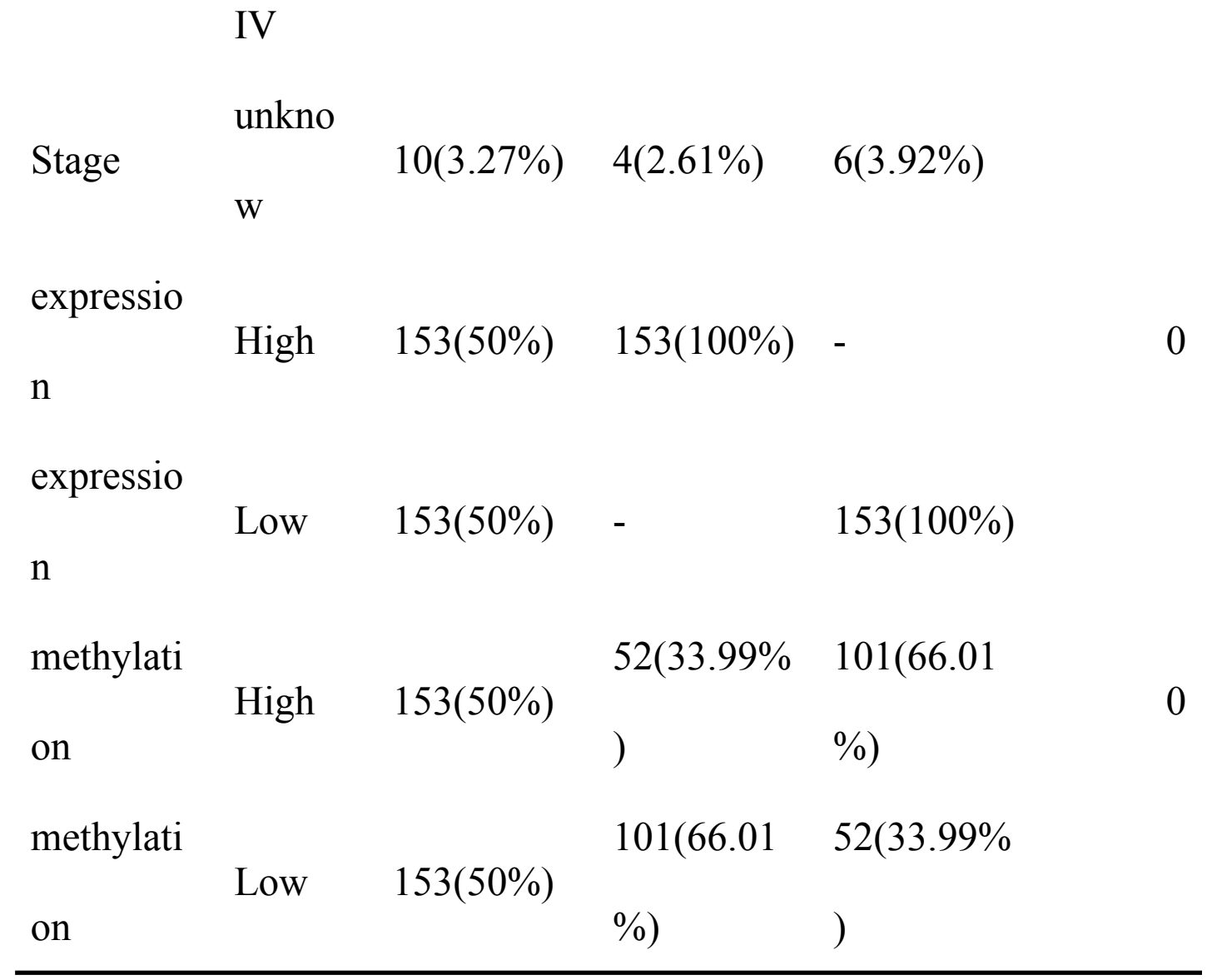

4 Abbreviations:T: Tumor, $N$ : Node, $M$ : Metastasis. 


\section{Figure 1}

Figure 1 Differential expression and survival analysis of the CRYAB gene.

(A) CRYAB mRNA expression in different cancers; red and blue represent downregulation and upregulation, respectively. (B) Comparative expression of CRYAB mRNA between colorectal tumor tissues and normal tissues; red and blue represent tumor and normal, respectively (statistical significance calculated by difference analysis, $* P<0.05$; $* * P<0.01$; *** $P<$ 0.001). (C) Human CRYAB expression levels in different cancer tissues and corresponding normal tissues. (D-F) The Kaplan-Meier survival curves of CRC patients with high and low CRYAB expression levels. (G) A meta-analysis with two data sets. High expression of CRYAB is significantly associated with poor OS. TE: estimated treatment effect; seTE: standard error of treatment estimate. 


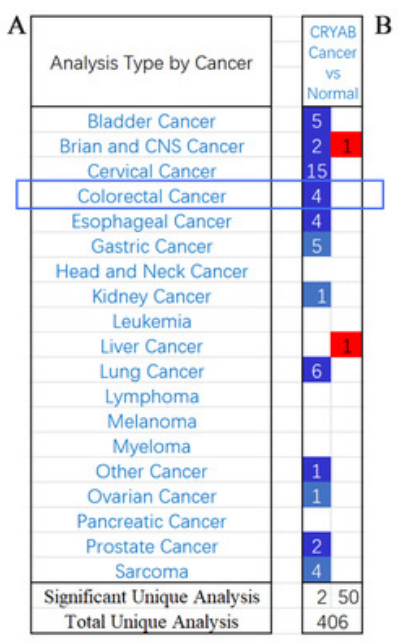

$\mathrm{C}$

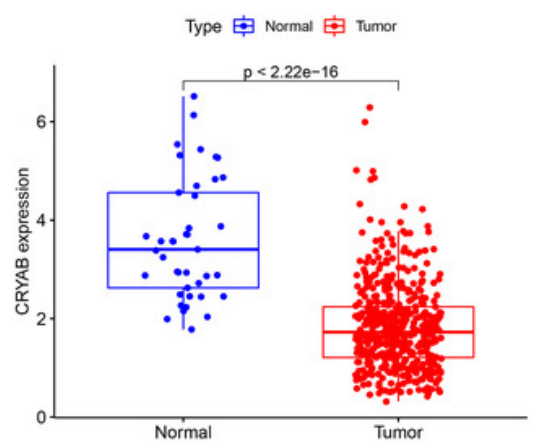

E

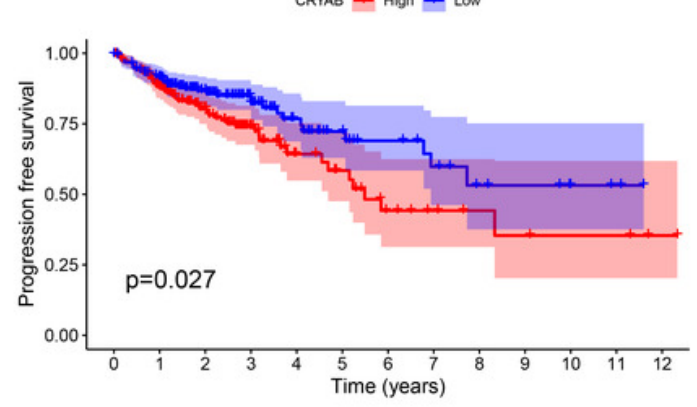

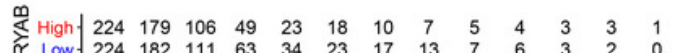

音 Low \begin{tabular}{|ccccccccccccc}
-224 & 182 & 111 & 63 & 34 & 23 & 17 & 13 & 7 & 6 & 3 & 2 & 0 \\
\hline 0 & 1 & $\dot{2}$ & $\dot{3}$ & $\dot{4}$ & $\dot{5}$ & $\dot{6}$ & $\dot{7}$ & $\dot{8}$ & $\dot{9}$ & 10 & 11 & 12
\end{tabular}

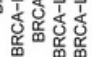

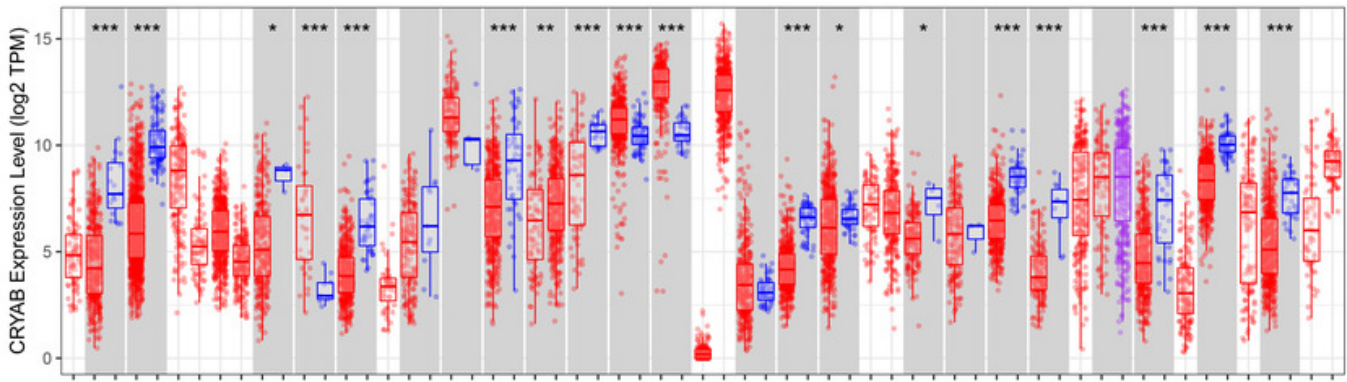

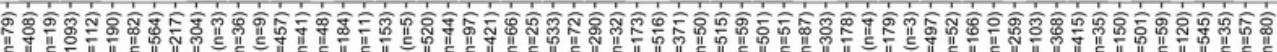

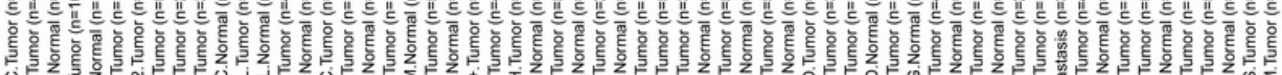

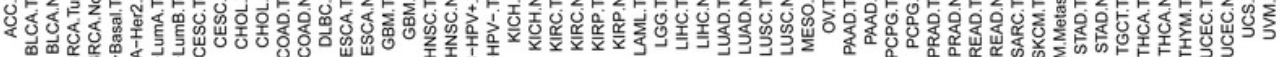

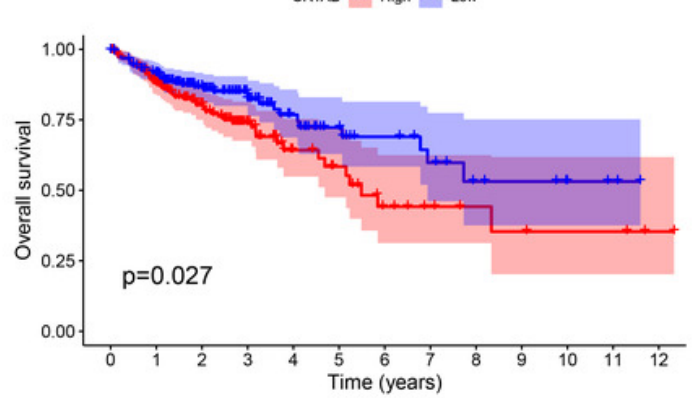

罢 Hight $224 \begin{array}{llllllllllll}2249 & 106 & 49 & 23 & 18 & 10 & 7 & 5 & 4 & 3 & 3 & 1\end{array}$ 竞 Low. \begin{tabular}{cccccccccccccc}
-224 & 182 & 111 & 63 & 34 & 23 & 17 & 13 & 7 & 6 & 3 & 2 & 0 \\
\hline 0 & 1 & $\dot{2}$ & $\dot{3}$ & $\dot{4}$ & $\dot{5}$ & $\dot{6}$ & $\dot{7}$ & $\dot{8}$ & $\dot{9}$ & 10 & 11 & 12
\end{tabular}

$\mathrm{F}$

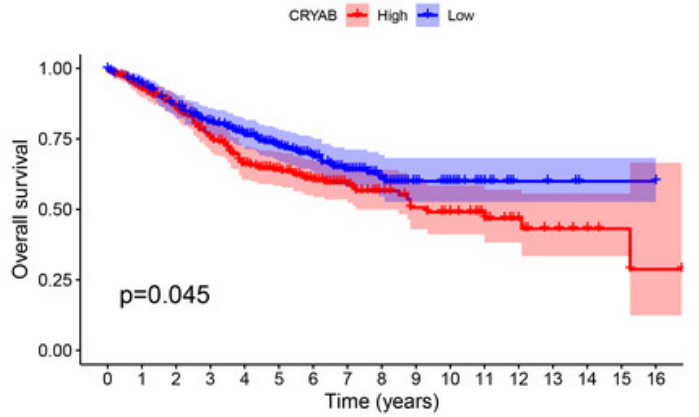

d Hight $289261228188145112906042322821 \quad 14 \quad 8 \quad 6 \quad 3 \quad 1$

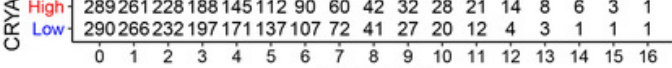
Time (years)

G

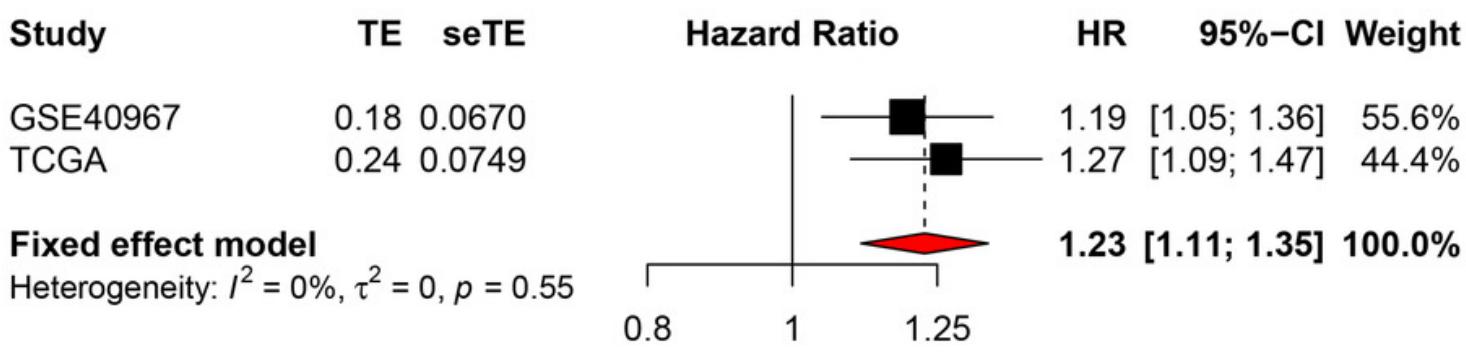




\section{Figure 2}

Figure 2 Representative IHC staining of CRYAB expression in colorectal cancer tissues and normal colorectal tissues.

Representative histopathological sections of (A-C) normal colorectal tissues and (D-F) colorectal cancer tissues stained with IHC. The colon section was incubated with HRP-labeled goat anti-pika universal antibody and stained with DAB (brown). Images are shown at 200x magnification (scale bar, $100 \mu \mathrm{m}$ ). IHC: immunohistochemistry. (G) We cut three sets of immunohistochemical sections, including three normal tissues and three cancer tissues, and tested the number of positive cells. 
A
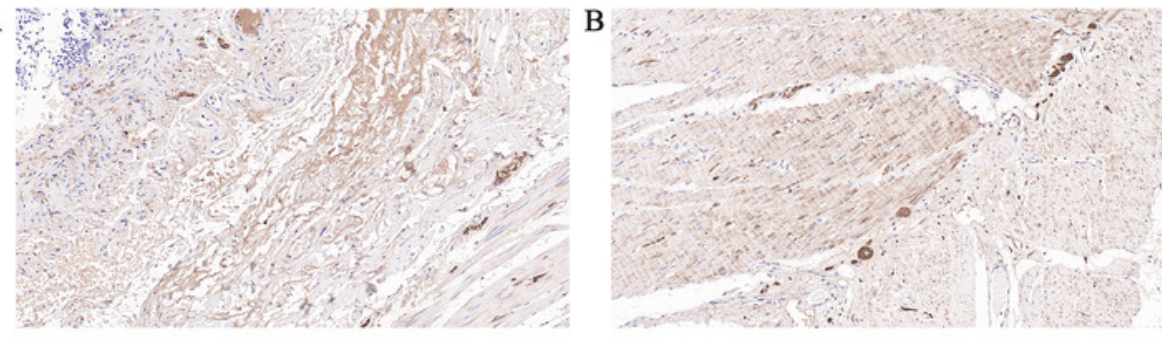

D
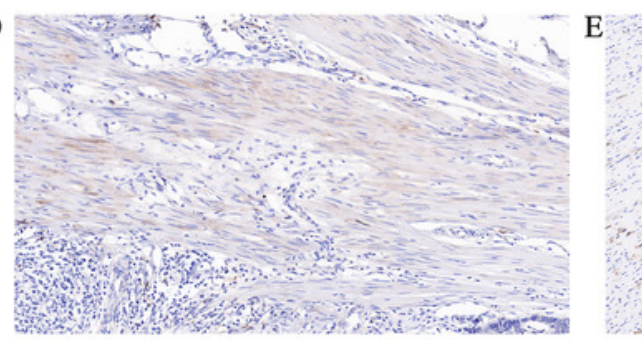
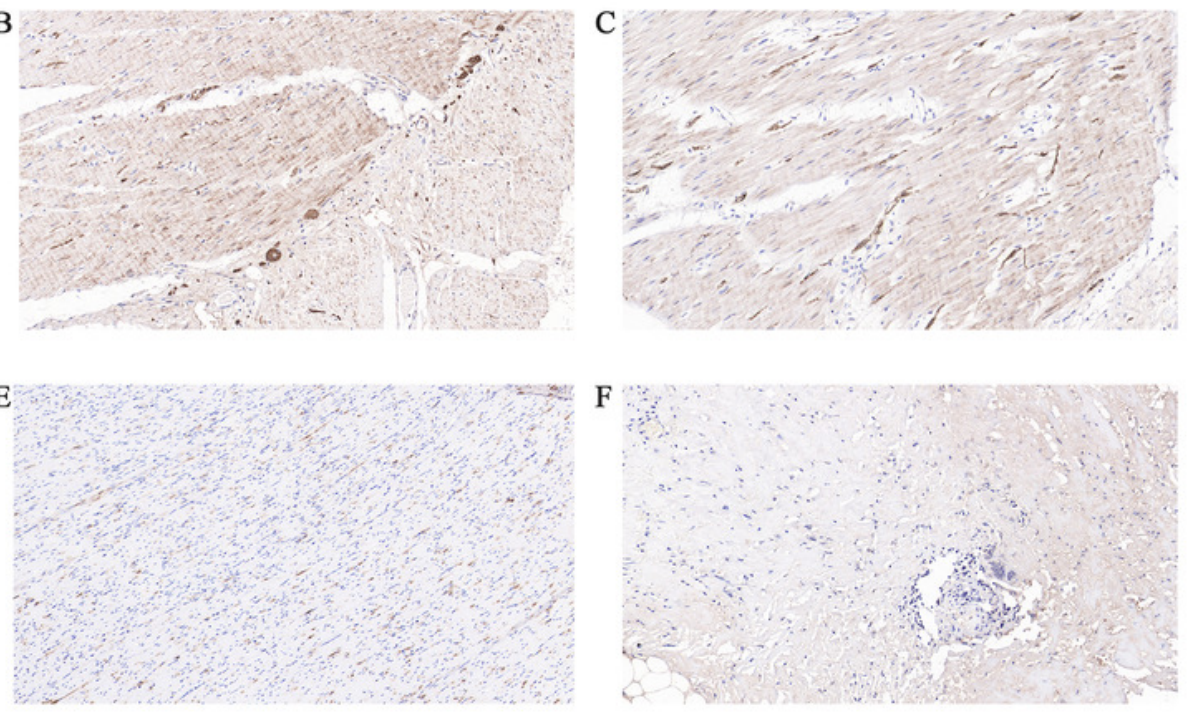

$\mathrm{F}$

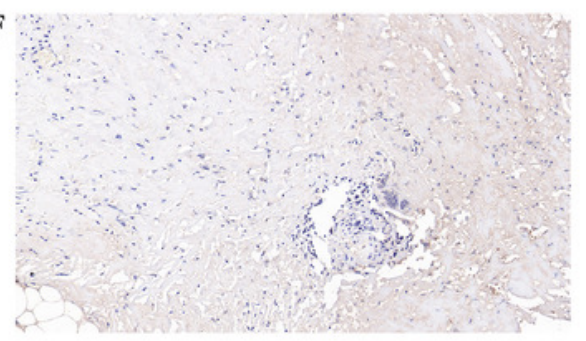

G CRYAB

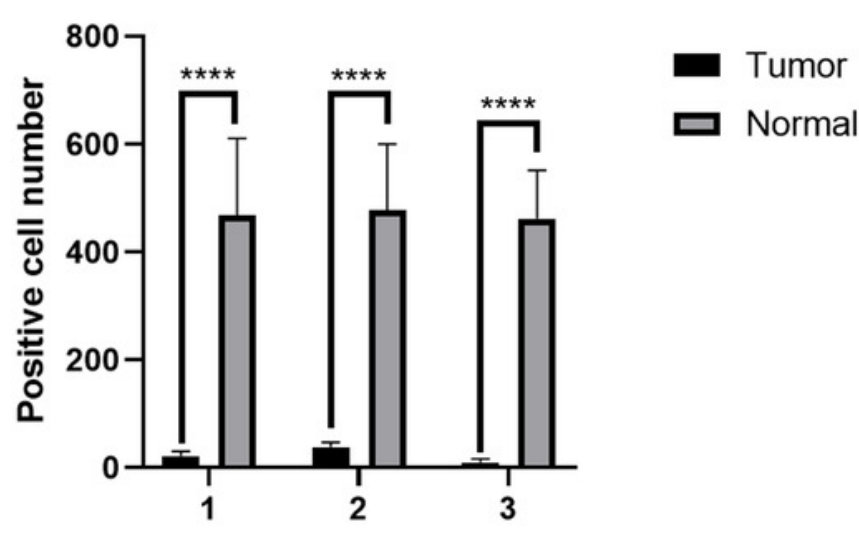


Figure 3

Figure 3 Relationship between the expression of CRYAB and clinicopathological characteristics of colorectal cancer patients.

(A-F) Box-plot showing the relation of CRYAB mRNA to (A) age, (B) gender, (C) distant metastasis, (D) lymph node metastasis, (E) tumor size, and (F) cancer stage.
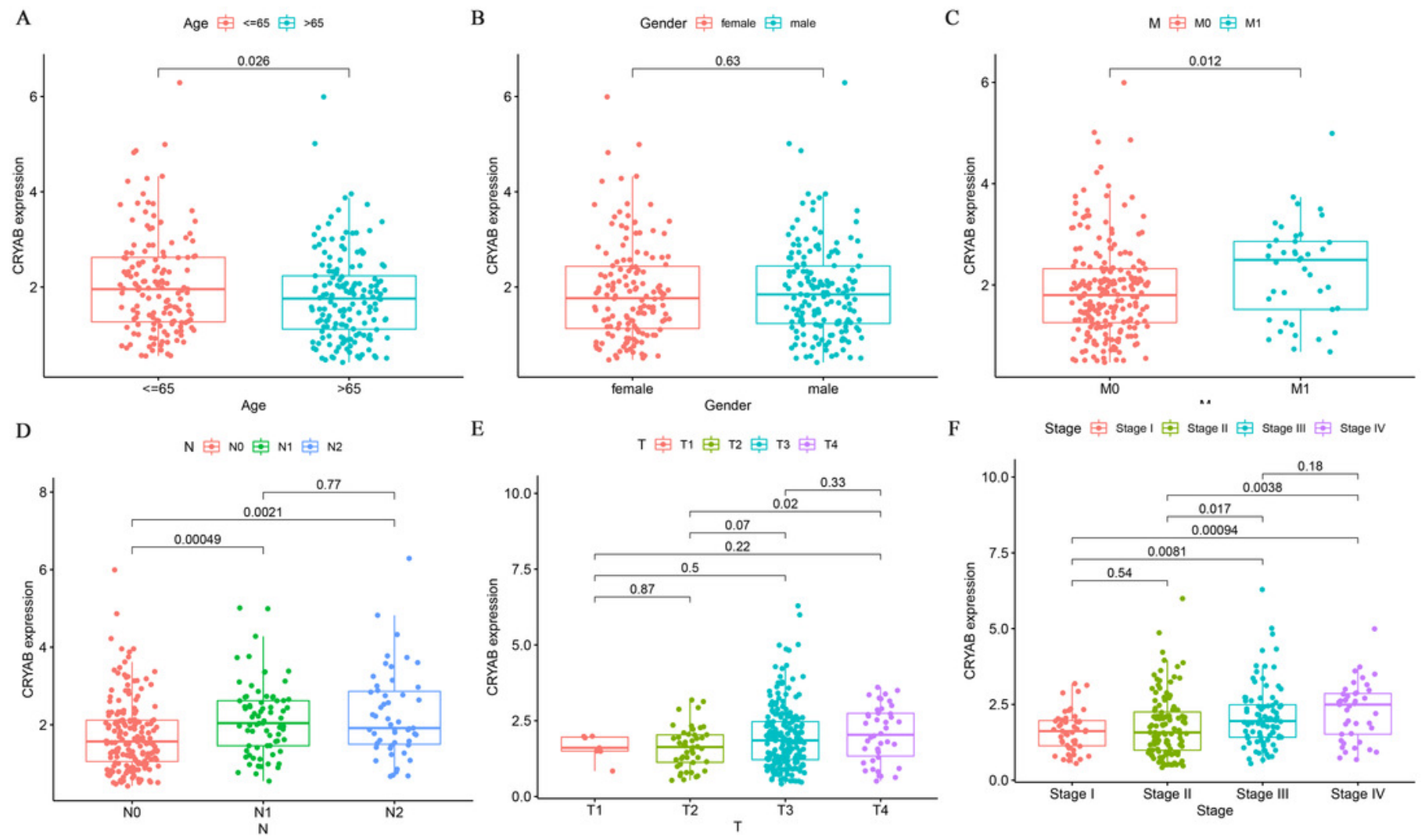

E
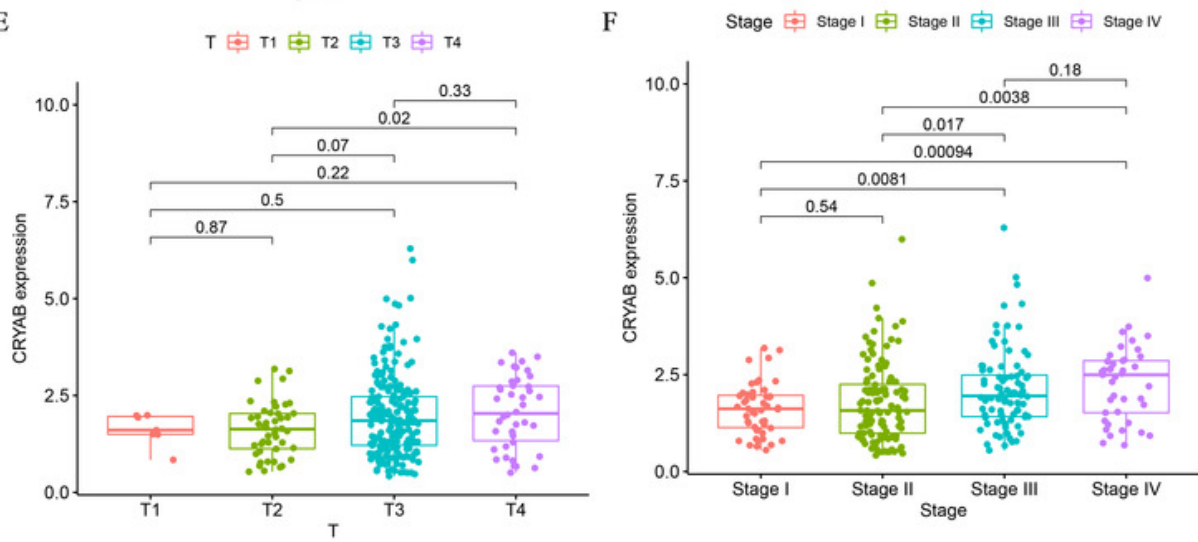
Figure 4

Figure 4 Correlation between CRYAB expression and immune infiltration in CRC.

Correlations between CRYAB expression and different immune cells: (A) neutrophils, (B) macrophages, (C) CD4+T cells, and (D) CD8+T cells. Correlations between CRYAB expression and different immune-related genes: (E) CD2, (F) CD2D, and (G) CD2E. 
A

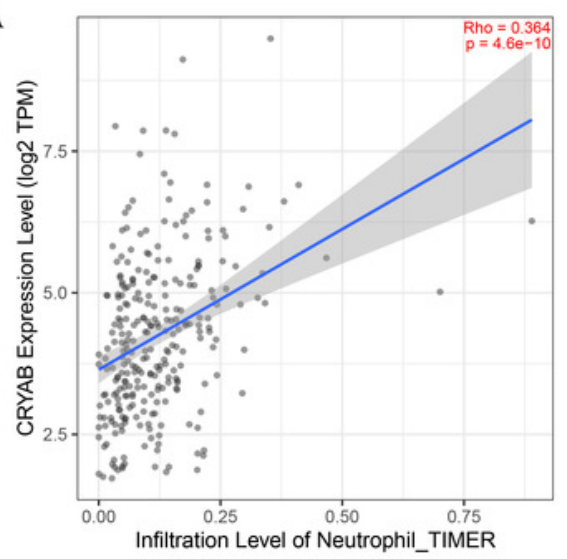

D

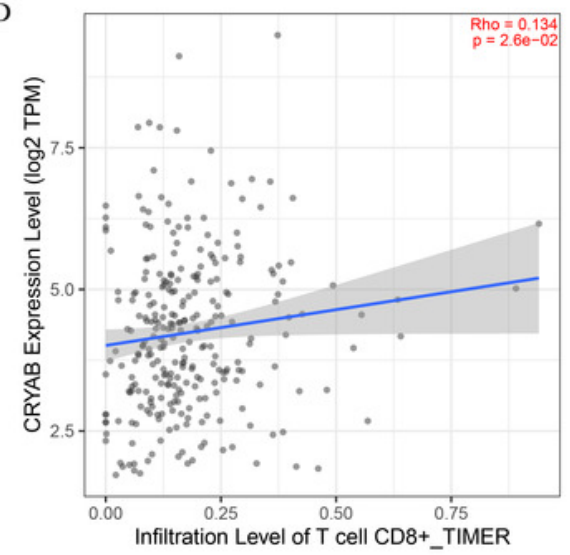

G

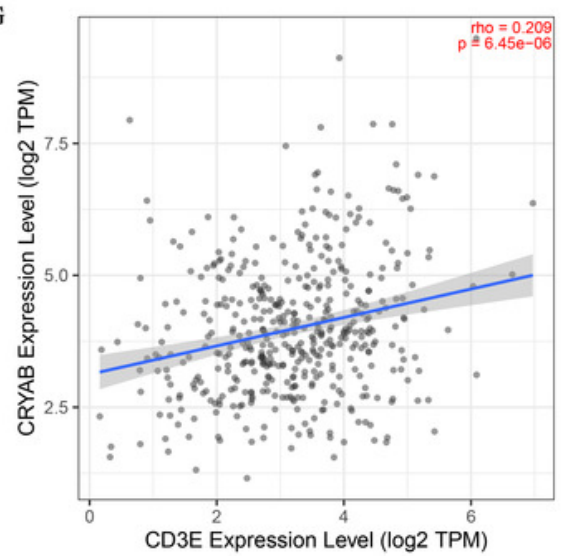

B

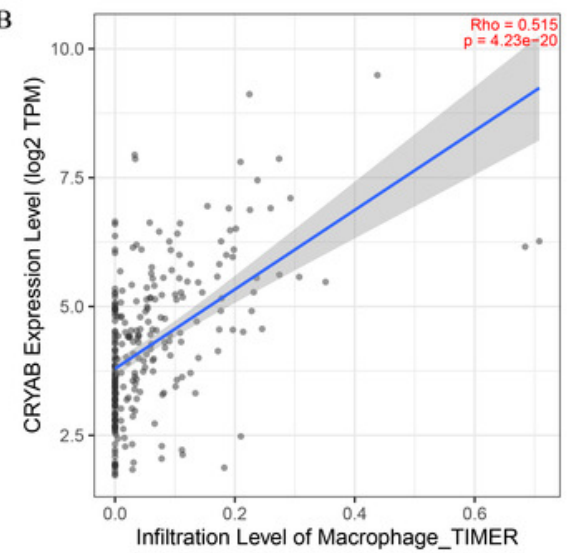

E

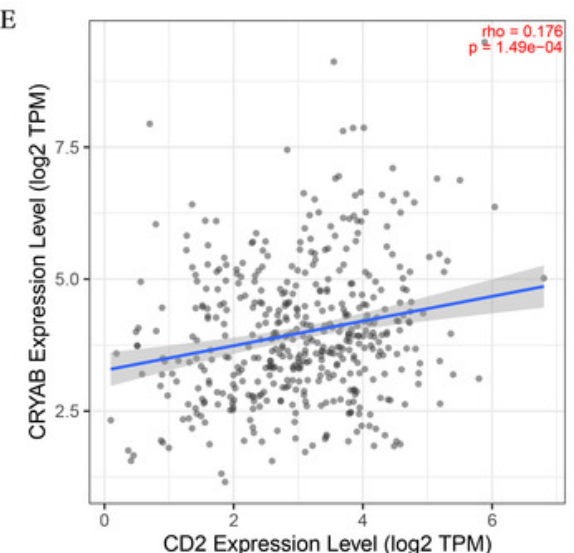

C

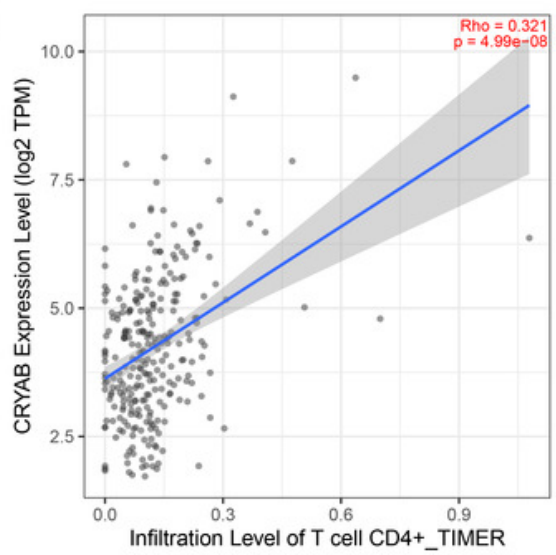

F

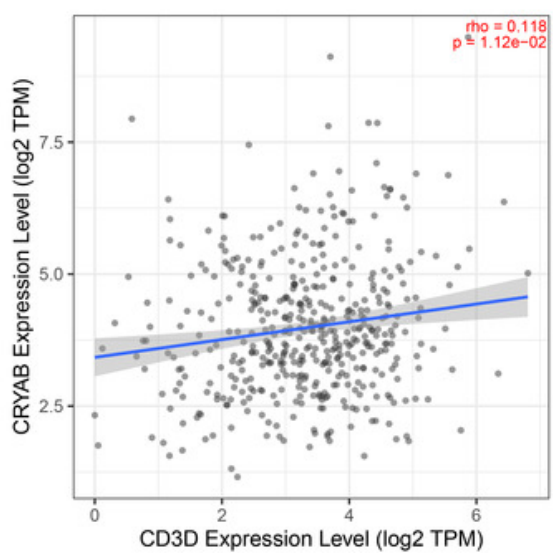


Figure 5

Figure 5 Correlation between CRYAB expression and genetic markers of M2-like macrophages.

(A, B) Correlation between CRYAB expression and TGFB1 in pan-carcinoma (A) and CRC (B).

(C, D) Correlation between CRYAB expression and MRC1 in pan-carcinoma (C) and CRC (D). $(E, F)$ Correlation between CRYAB expression and CD163 in pan-carcinoma (E) and CRC (F). $P$ $<0.05$ indicates statistical significance. 
A

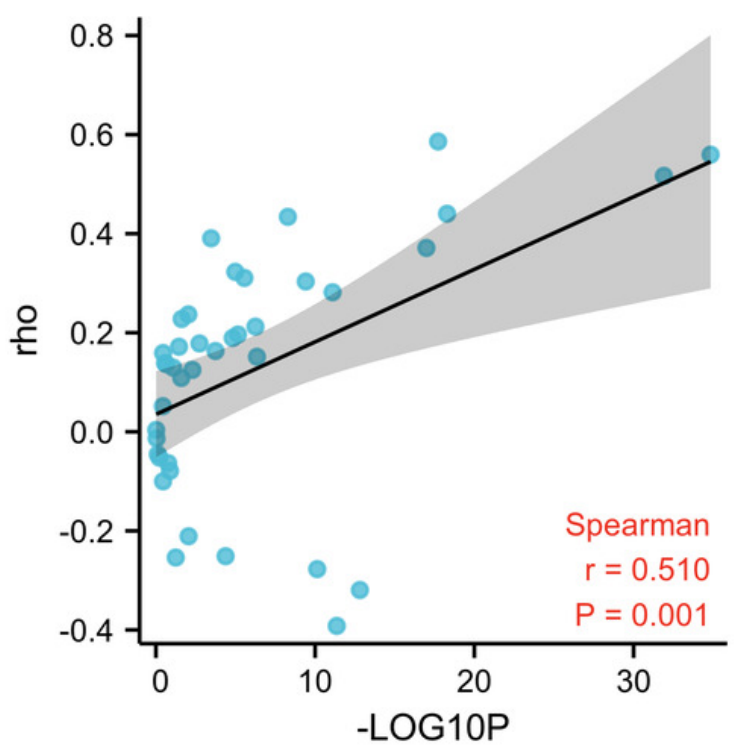

C

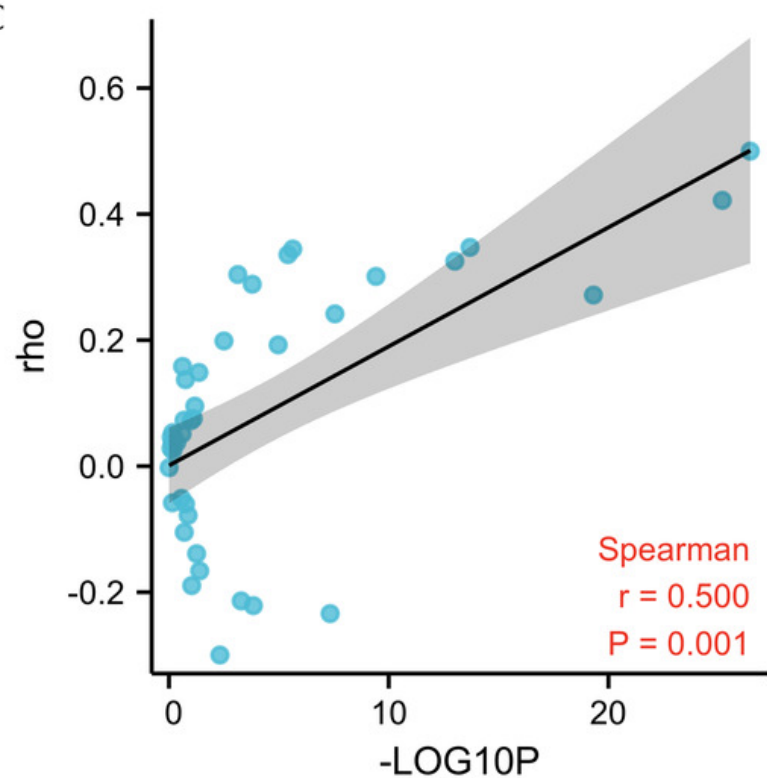

E

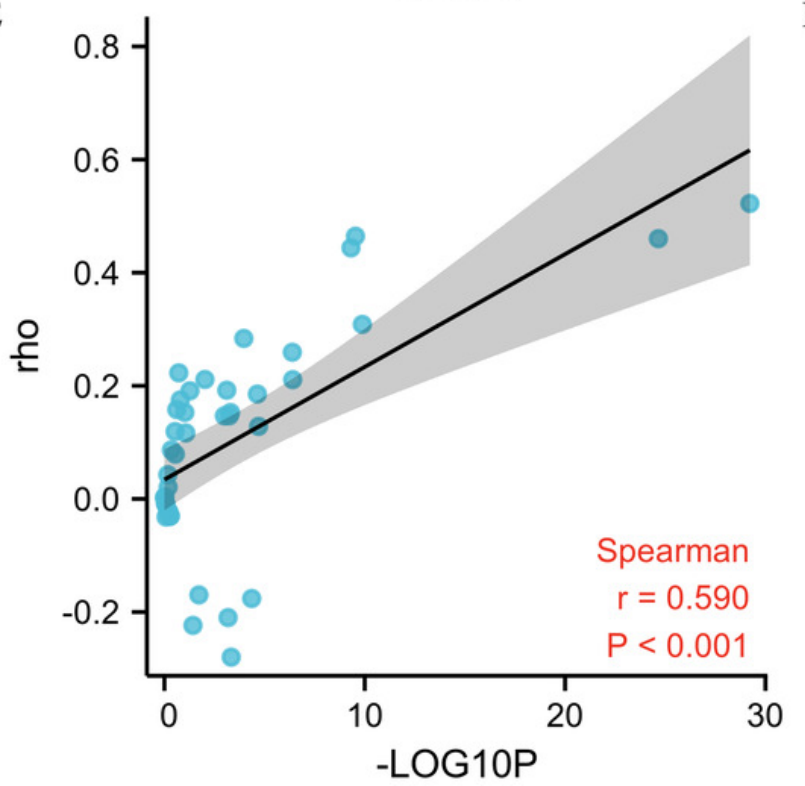

B

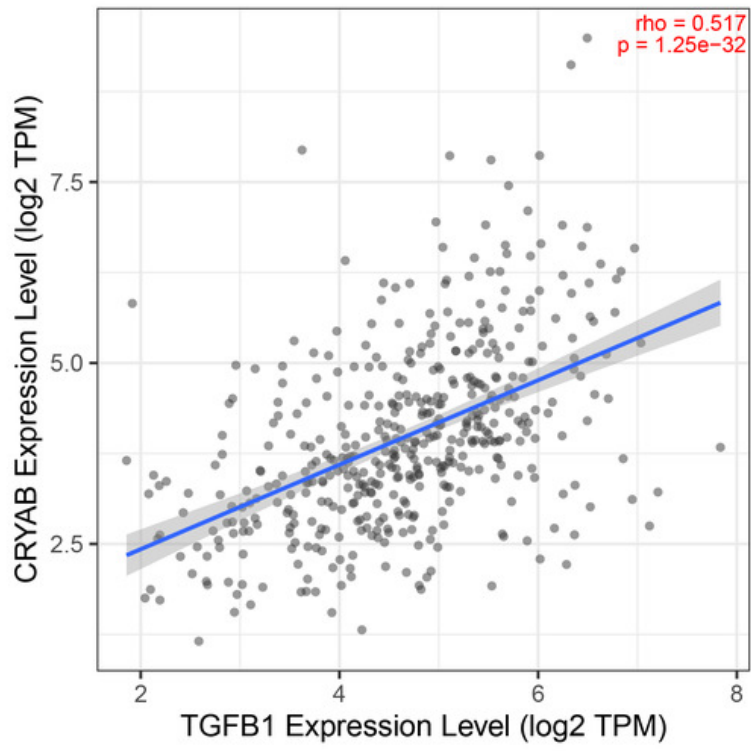

D

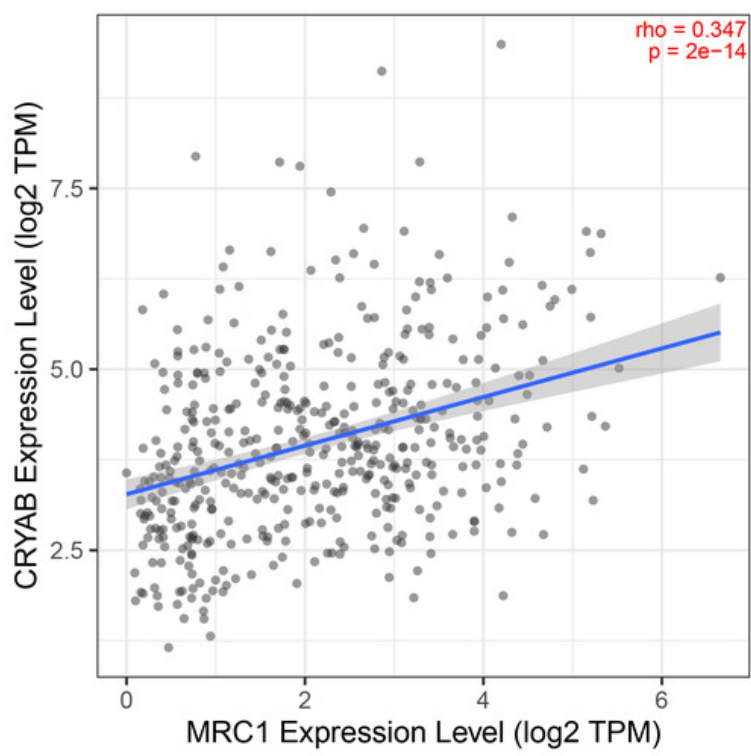

F

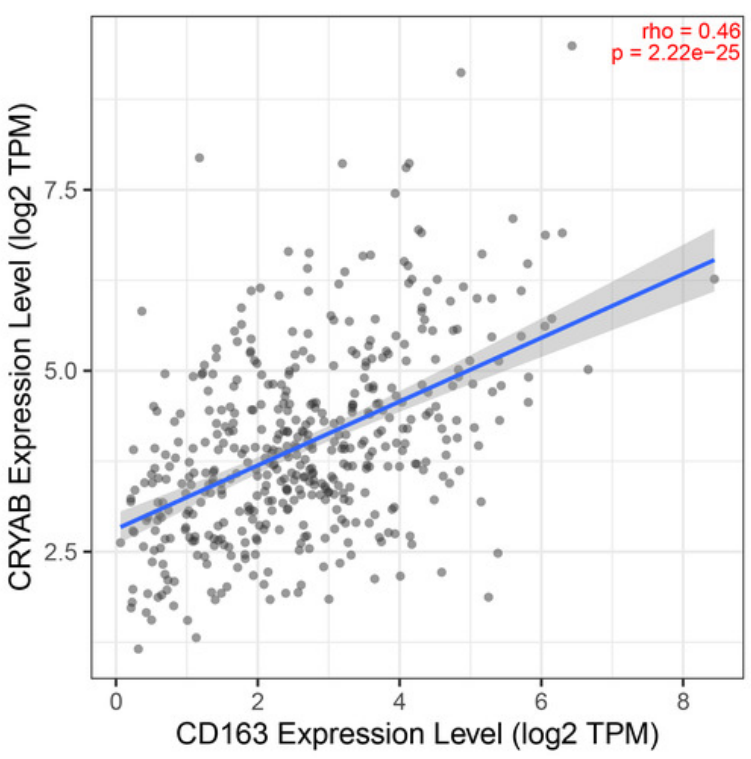


Figure 6

Figure 6 Relationship between CRYAB methylation and CRYAB gene expression in CRC

(A) Methylation level of each methylation site (B-C) Correlation between CRYAB gene methylation site and CRYAB gene expression. 
A

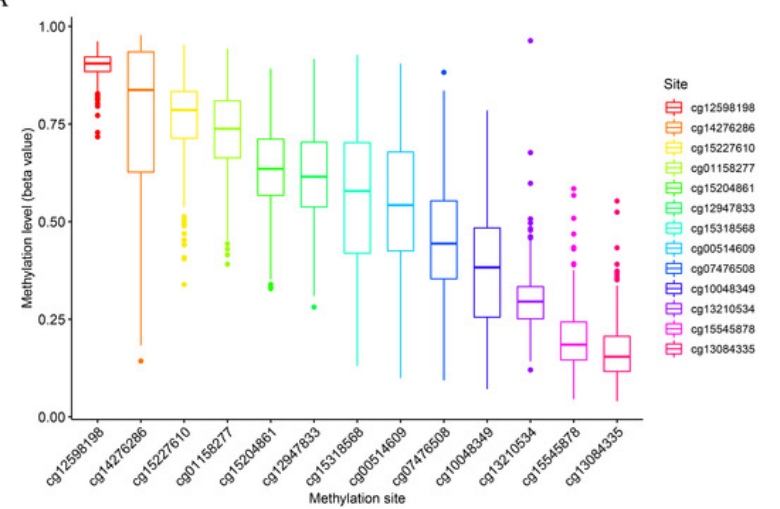

B
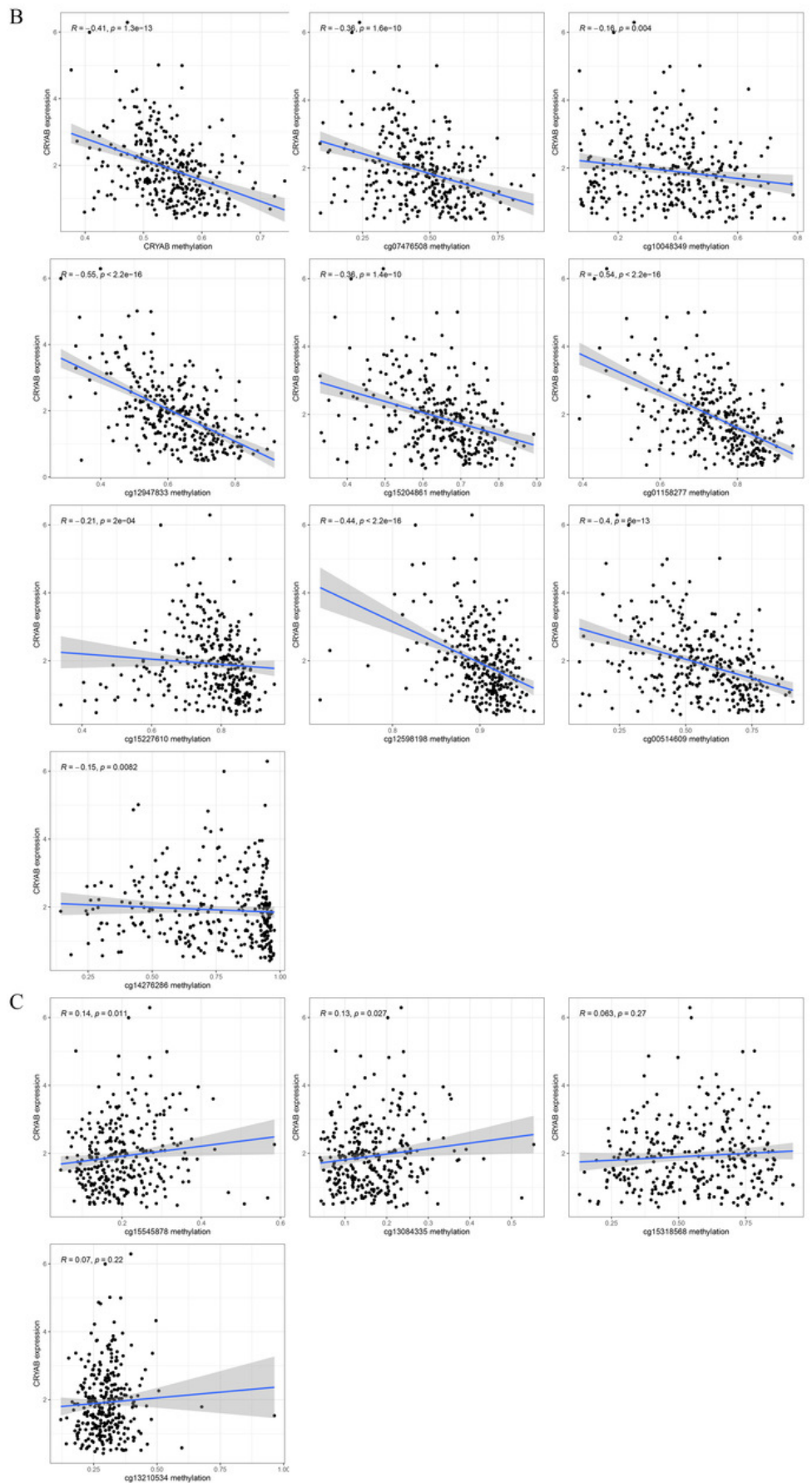

Peer] reviewing PDF | (2021:03:59504:2:1:NEW 2 Nov 2021) 
Figure 7

Figure $7 \mathrm{GO}$ and KEGG enrichment analyses.

(A) Gene enrichment in three different GO functions and (B) KEGG pathways were respectively ranked byp-value and gene enrichment count.

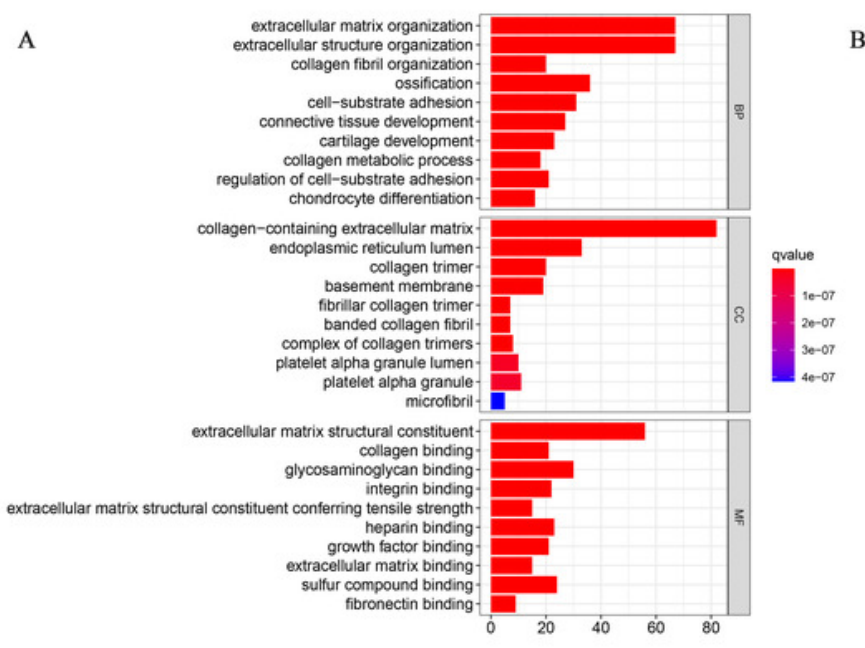

C

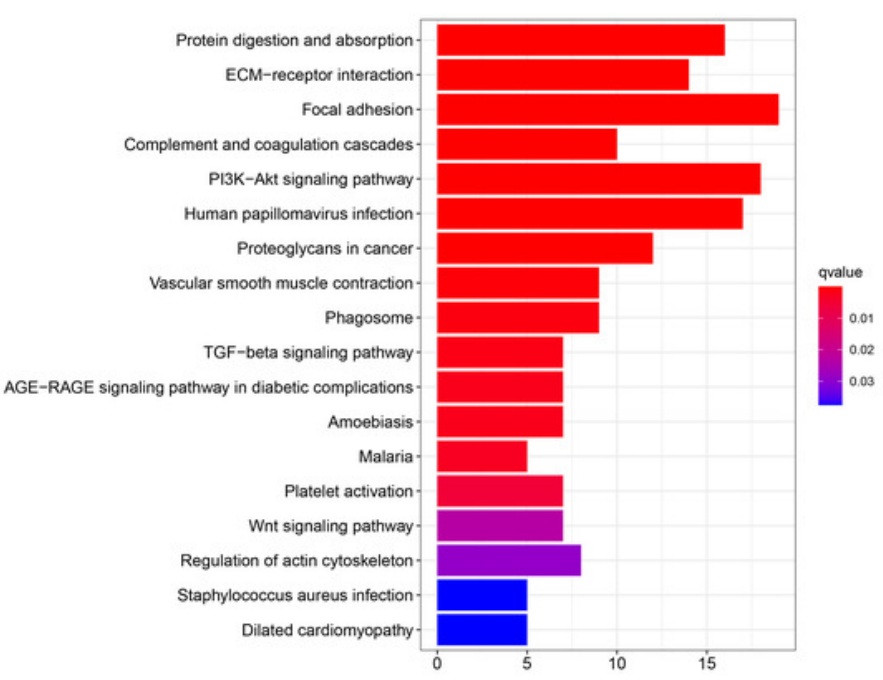

D
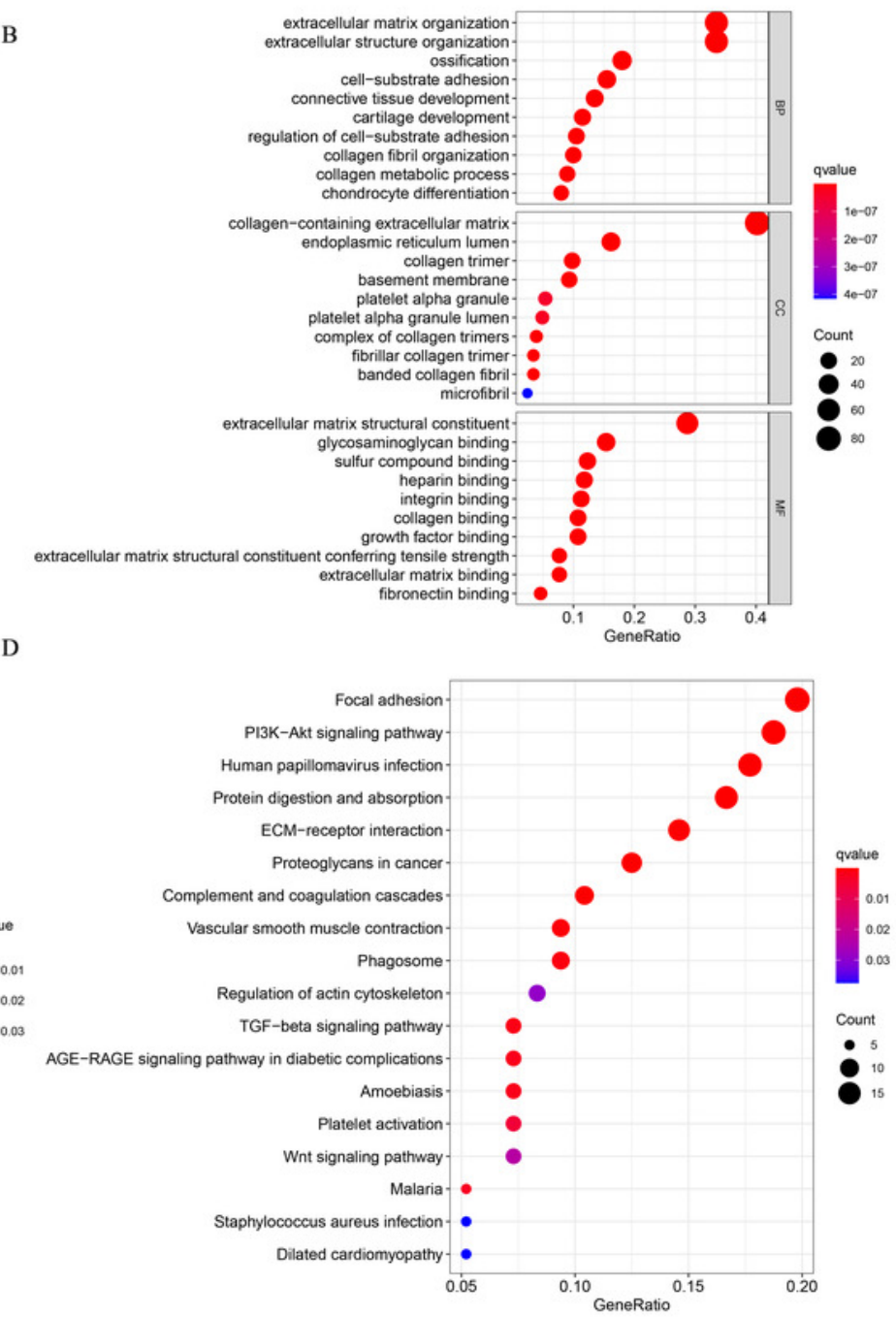